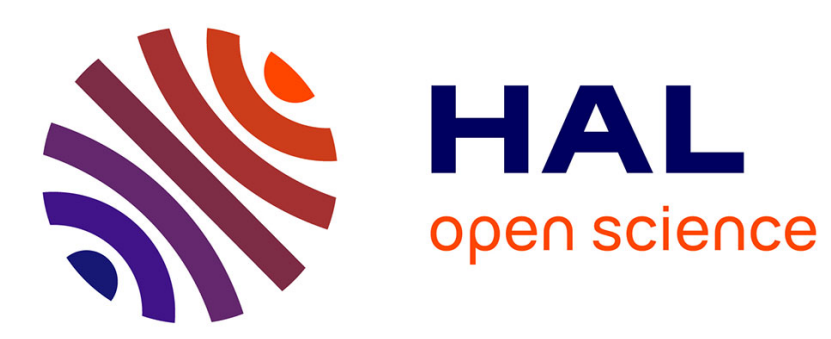

\title{
Les salariés de plus de 50 ans et l'informatique: une comparaison public-privé
}

Danièle Guillemot, Aurélie Peyrin

\section{To cite this version:}

Danièle Guillemot, Aurélie Peyrin. Les salariés de plus de 50 ans et l'informatique: une comparaison public-privé. Economie et Statistique / Economics and Statistics, 2011, 437, p. 3-28. halshs-01102397

\section{HAL Id: halshs-01102397 \\ https://shs.hal.science/halshs-01102397}

Submitted on 13 Jan 2015

HAL is a multi-disciplinary open access archive for the deposit and dissemination of scientific research documents, whether they are published or not. The documents may come from teaching and research institutions in France or abroad, or from public or private research centers.
L'archive ouverte pluridisciplinaire HAL, est destinée au dépôt et à la diffusion de documents scientifiques de niveau recherche, publiés ou non, émanant des établissements d'enseignement et de recherche français ou étrangers, des laboratoires publics ou privés. 
TRAVAIL - EMPLOI

\title{
Les salariés de plus de 50 ans et l'informatique : une comparaison public-privé
}

\author{
Danièle Guillemot* et Aurélie Peyrin**
}

Dans les années 1990 et 2000, de nombreux travaux ont mis en évidence l'impact négatif, sur l'emploi des salariés âgés, des changements techniques et organisationnels opérés par les entreprises. Cet effet défavorable du changement a été attribué notamment à un défaut d'adaptabilité des seniors aux technologies informatiques, conduisant à dévaloriser les compétences acquises au cours de la carrière. De plus, la formation à la maîtrise de ces nouvelles techniques est souvent considérée comme moins efficace et moins rentable lorsqu'elle bénéficie à des seniors plutôt qu'à des salariés plus jeunes. Cette question de la capacité des salariés âgés à s'adapter aux outils informatiques est actualisée ici à partir de l'enquête Changement organisationnel et informatisation (COI) de 2006, dont le champ est étendu aux activités administratives de l'État. À la différence des entreprises, la fonction publique de l'État maintient en emploi l'ensemble de ses agents titulaires, quelle que soit leur capacité supposée d'adaptation aux changements. Si les salariés âgés étaient, en général, peu adaptables aux outils informatiques, l'usage de ces outils devrait décliner avec l'âge, au sein de la fonction publique de l'État plus encore que dans les secteurs marchands. Les résultats empiriques montrent au contraire que l'usage de l'informatique se maintient après 50 ans dans la fonction publique alors qu'il décline avec l'âge dans le privé. L'État forme en effet ses agents à l'informatique plus souvent et plus longuement que le secteur privé, ces formations bénéficiant, au cours de leur carrière, à une majorité de seniors. L'entraide entre collègues apparaît également favorable à l'accès des plus âgés aux outils technologiques récents. Lorsque l'activité le requiert et que l'organisation le prévoit, les salariés âgés de plus de 50 ans peuvent donc s'adapter aux nouveaux outils informatiques. 
$\grave{\mathbf{A}}$ partir d'enquêtes réalisées dans les années 1990 en France (1) et à l'étranger, de nombreux travaux ont établi l'existence d'un « biais technologique »défavorisant les salariés les plus âgés lorsque les entreprises s'informatisent. Deux mécanismes de sélection par l'âge s'additionnent. D'une part, les salariés âgés (mais aussi les salariés très jeunes) sont relativement peu nombreux dans les entreprises les mieux équipées en informatique de pointe, soit que ces entreprises se séparent d'une partie de leurs seniors, soit qu'une structure de la main-d'œuvre concentrée sur les âges intermédiaires favorise la modernisation technologique (Ananian et Aubert, 2006 ; Aubert et al., 2006 ; Diaye et al., 2006). D'autre part, les entreprises sélectionnent parmi leurs salariés ceux qui accèdent aux outils informatiques (Gollac et al., 2000 ; Moatty, 1993 ; Friedberg, 2003 ; HamonCholet et Vinck, 2004) : si les seniors qui restent en emploi sont a priori, du point de vue des employeurs, plus adaptables aux changements technologiques que ceux qui ont été évincés, leur poste de travail est cependant moins fréquemment informatisé que celui des salariés plus jeunes. Cette sélection s'appuierait sur des représentations sociales courantes concernant l'obsolescence des acquis de l'expérience des seniors et leurs difficultés d'apprentissage à de nouveaux outils (Minni et Topiol, 2003), alors que les plus jeunes sont déjà formés à l'informatique. Les salariés les plus âgés ont également moins accès à la formation, cet investissement pouvant ne pas apparaître rentable au regard du nombre d'années avant leur départ en retraite du point de vue de l'employeur comme de celui de l'employé.

Le moindre accès des seniors à l'informatique, mesuré dans les années 1990, pourrait cependant n'avoir été qu'un phénomène transitoire. Constatant une nette baisse des taux d'utilisation après 45 ans à partir de l'enquête Techniques et organisation du travail (TOTTO) 1991, Moatty (1993) a émis l'hypothèse d'un effet de génération qui frapperait les salariés en deuxième partie de carrière, au moment de l'introduction des ordinateurs dans les entreprises. Or, cette génération est aujourd'hui majoritairement retraitée, et on peut penser que les seniors les plus en difficulté face à l'outil informatique (s'ils ne sont pas encore retraités) font partie de ceux qui, chômeurs, préretraités ou en invalidité, sont écartés du marché du travail. Les distinctions entre salariés peuvent cependant être renouvelées avec l'arrivée de nouveaux outils (Gollac et Kramarz, 2000) : plutôt que le simple accès aux ordinateurs, c'est sans doute désormais l'utilisation d'équipements plus sophistiqués, d'applications variées, ou le degré de maîtrise des outils, qui distingue les salariés entre eux. La mise à l'écart des seniors (ou de ceux d'entre eux qui seraient les moins adaptables aux nouvelles technologies) par les employeurs pourrait donc en théorie persister, qu'elle se traduise par l'évincement de l'emploi ou bien l'affectation à des postes ne nécessitant qu'une utilisation banale de l'informatique.

L'enquête Changement organisationnel et informatisation (COI) de 2006 (cf. encadré 1) apporte des éclairages nouveaux sur ces questions. Sur le champ des salariés des entreprises d'au moins 20 salariés des secteurs marchands et sur celui des agents de la fonction publique de l'État (2) (hors enseignants, militaires et magistrats), l'enquête apporte de nombreuses informations sur l'usage de l'informatique, de la saisie sur terminal mono-tâche aux pratiques nomades ou collaboratives et à la dématérialisation des procédures, mais aussi sur certaines dimensions du travail qui peuvent en permettre la maîtrise, comme la formation aux outils ou l'entraide entre salariés. De plus, l'élargissement du champ de l'enquête à la fonction publique de l'État permet de renouveler les registres interprétatifs habituels. Dans la fonction publique de l'État, le statut protège en effet les agents des licenciements quels que soient leur âge et leur capacité supposée d'adaptation aux changements (3) ; la proportion de salariés âgés y est d'ailleurs plus élevée que dans les autres secteurs. La gestion de la main-d'œuvre dans la fonction publique de l'État présente des caractéristiques propres au modèle du marché interne (Behaghel et Gautié, 2006), où l'accumulation d'un capital humain spécifique, via la formation interne, joue un rôle central. Au sein de ces formes institutionnelles, les relations de long terme entre les salariés et leur employeur sont privilégiées, et de ce fait, l'ancienneté moyenne est élevée. Mais les nouvelles technologies tendraient à dévaloriser le capital humain spécifi-

\footnotetext{
1. Notamment les enquêtes Changement organisationnel et informatisation (COI) 1997 et Relations professionnelles et Négociations d'entreprise (REPONSE) 1998

2. Par commodité, nous utilisons par la suite les termes de "public » pour désigner la fonction publique de l'État d'une part, et " privé » ou " entreprises » les entreprises des secteurs marchands (dans lesquels des entreprises de statut public, comme la Poste ou la SNCF sont classées) d'autre part.

3. L'âge de la retraite est cependant plus précoce dans certains métiers de la fonction publique liés à la sécurité comme les policiers ou le personnel pénitentiaire, mais ces sorties précoces de l'emploi ne sont pas dues à une sélection opérée par l'employeur. L'utilisation de l'informatique est moins répandue dans ces métiers qu'ailleurs, ce qui peut contribuer à relever le taux d'utilisation moyen des plus de 50 ans. La problématique de l'article n'est cependant pas affectée par ce phénomène.
} 
que (Caroli, 2000), ce qui est une des explications avancées au déclin des marchés internes au sein des grandes entreprises (Gautié, 2004). Or, malgré le poids important des seniors, l'État s'est fortement modernisé au cours des vingt dernières années, mettant notamment en place des progiciels de gestion intégrée et généralisant l'e-administration (Alcaud et Lakel, 2004 ; Lau, 2004). Aujourd'hui, la plupart des agents est amenée à utiliser un ordinateur (Guillemot et Peyrin, 2009).
On fait l'hypothèse que, dans le cas de l'État où le maintien dans l'emploi est garanti par le statut, l'organisation doit se moderniser tout en s'adaptant à la main-d'œuvre en place. Des régulations spécifiques, comme une formation soutenue aux outils tout au long de la carrière, doivent alors être mises en œuvre pour permettre la généralisation de l'informatique. L'entraide entre salariés de différentes générations peut également jouer un rôle dans l'adaptation aux nouvelles technologies.

\section{Encadré 1 \\ LA MESURE DE L'INFORMATISATION DU TRAVAIL À TRAVERS LES ENQUÊTES CHANGEMENTS ORGANISATIONNELS ET INFORMATISATION ET CONDITIONS DE TRAVAIL}

Les données et le champ de l'enquête Changements organisationnels et informatisation (COI) 2006

COI 2006 est une enquête auprès des employeurs et de leurs salariés. Les employeurs sont interrogés sur les changements d'organisation et les outils informatiques et de gestion adoptés par leur entreprise ou administration. Les salariés sont questionnés sur leur travail et les outils informatiques qu'ils utilisent. Nous utilisons cependant ici presqu'exclusivement le volet " salariés ". En effet, les volets " employeurs" des secteurs marchands et de la fonction publique de l'État ont été réalisés à des dates différentes, avec des questionnaires spécifiques. Ils comportent cependant des questions communes, dont celles relatives à l'utilisation par l'entreprise ou l'administration de divers outils informatiques, mobilisées dans cette étude.

Le champ de l'enquête auprès des employeurs dans les secteurs marchands a été défini par un règlement européen, pour des indicateurs harmonisés sur l'équipement en TIC des entreprises II inclut les entreprises d'au moins 10 salariés des secteurs marchands non agricoles, y compris les secteurs financiers et recherche-développement (habituellement exclus des enquêtes de l'Insee auprès des entreprises), mais ne prend pas en compte les services aux particuliers ; les hôtels-restaurants et les médias font toutefois partie du champ de l'enquête. Le champ de l'enquête dans la fonction publique de l'État est lui aussi particulier : il comprend les directions d'administrations centrales et déconcentrées des ministères (la taille minimale est de 10 agents), à l'exception de celui de la Défense, et hors établissements publics.

Les entreprises et administrations ont été échantillonnées dans un premier temps, puis les salariés ont ensuite été tirés au sort parmi un sous-échantillon d'employeurs d'au moins 20 salariés ayant répondu au premier volet. Au sein de la fonction publique de l'État, tous les agents, fonctionnaires ou contractuels, du champ de l'enquête "employeurs » font partie du champ "salariés ", à l'exception notable des enseignants et magistrats (mais les autres personnels des ministères concernés sont inclus dans le champ). L'extension de l'enquête $\mathrm{COI}$ à la fonction publique de l'État a en effet été conçue dans un objectif de comparaison avec les secteurs marchands. Compte tenu de la taille de l'échantillon, ces professions très spécifiques, qui ne peuvent guère être "comparées " à celles exercées dans les secteurs marchands, ont été écartées. L'échantillonnage de l'enquête $\mathrm{CO}$ l dans la fonction publique de l'État déforme alors la structure socioprofessionnelle de ses agents : au niveau agrégé, la suppression des enseignants entraîne une forte diminution de la part totale des cadres, au profit des catégories employés et ouvriers qui représentent $54 \%$ du nouvel échantillon, soit près du double de leur part au sein de l'ensemble des agents de l'État (cf. graphique). L'échantillonnage n'affecte en revanche pas la distribution selon le sexe, mais accroît la part - déjà importante en comparaison des salariés du privé - des "seniors " dans la fonction publique de l'État (Guillemot et Peyrin, 2008). Au total, environ 15000 salariés ont été interrogés dans les secteurs marchands et 1200 dans la fonction publique de l'État.

\section{Distribution des salariés par catégorie socioprofessionnelle et par secteur}

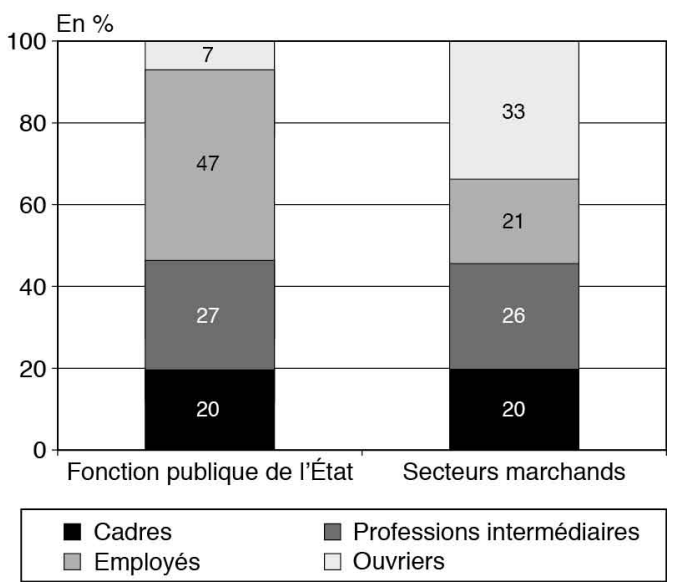

Champ : pour la fonction publique de l'État hors enseignants, magistrats, agents du ministère de la Défense et des établissements publics. Pour les secteurs marchands : salariés des entreprises marchandes non agricoles de plus de 20 salariés. Source : COI 2006, Statistique publique, DGAFP-Dares-CEE. 


\section{Une comparaison de la fonction publique de l'État avec l'ensemble des secteurs marchands}

É tablir une comparaison entre les salariés d'entreprises des secteurs marchands et les agents de la fonction publique de l'État nécessite de prendre quelques précautions. $\mathrm{La}$ fonction publique de l'État représente en effet un secteur à part, pour plusieurs raisons : l'activité des salariés répond en grande partie aux missions régaliennes de l'État et les salaires sont financés par l'impôt ; le statut général des fonctionnaires de l'État garantit l'emploi de la grande majorité des agents, dont il règle également le déroulement de la carrière.

Les réformes de la fonction publique de l'État au fil des dernières décennies s'inscrivent cependant dans une démarche de rapprochement avec le secteur marchand, notamment au travers de modalités de gestion et d'outils communs aux deux univers (Bezes, 2009). L'extension de l'enquête COI 2006 à la fonction publique de l'État repose ainsi sur l'hypothèse que les changements dans le secteur public sont en partie réalisés avec des outils de gestion et des outils informatiques adoptés également par les gran- des organisations du secteur marchand ; la diffusion de ces outils est donc une opportunité pour observer les spécificités et les convergences des organisations et du travail de chacun des secteurs (cf. encadré 1).

\section{Comparer le travail dans les entreprises marchandes et dans la fonction publique de l'État?}

Si les relations d'emploi sont singulières dans le public, la nature quotidienne de l'activité, notamment dans les filières administratives de la fonction publique de l'État, peut-elle être rapprochée de celle que connaissent les salariés de certains secteurs marchands, notamment dans les services? Une part importante de l'activité de la fonction publique de l'État consiste à enregistrer, traiter, échanger et stocker de l'information : sur les citoyens justiciables, contribuables ou bénéficiaires ; sur les entreprises ou l'espace public ; sur son propre budget ou ses agents, etc. Toutes ces activités de type administratif, qui s'appuient sur un outillage informatique puissant, sont présentes dans la plupart des entreprises, avec des poids divers. Comme dans certains secteurs du privé, tels les banques et assurances, ce sont des activités centrales pour l'administration publique, alors qu'il s'agit ailleurs de fonctions « support » (gestion, comptabilité,

Encadré 1 (suite)

La croissance de l'informatisation mesurée par les enquêtes Conditions de travail (CDT) de la Dares

Le champ de l'enquête $C D T$ est plus complet que celui de COI, mais il ne permet pas de distinguer correctement la fonction publique de l'État des fonctions publiques territoriale et hospitalière. Les travaux d'expertise (en cours) du service statistique ministériel de la fonction publique montrent par exemple que les enseignants titulaires sont parfois affectés de façon erronée à un employeur public territorial ou privé. Quoi qu'il soit partiel par construction, l'échantillonnage de $\mathrm{CO}$ propose ainsi une meilleure adéquation avec le champ de la fonction publique de l'État tel que défini dans le tableau de synthèse publié chaque année dans le

Rapport annuel sur l'état de la fonction publique. Par ailleurs, l'enquête $C D T$ détaille moins la question de l'utilisation de l'informatique et de la formation aux outils que l'enquête $\mathrm{COI}$.

Cette source permet cependant d'estimer la croissance de l'informatisation entre 1991 et 2005, dans tous les secteurs. Elle a été particulièrement massive entre 1991 et 1998 (cf. tableau). Le taux d'utilisation de l'informatique au travail des agents de l'administration publique, initialement proche de celui de l'ensemble des entreprises (respectivement $32 \%$ et $33 \%$ en 1991), a dépassé l'ensemble des entreprises dès 1998 (60\% contre $49 \%$ ) et confirmé son « avance » en 2005 (67\% contre $58 \%$ ).

Taux d'utilisation d'un ordinateur au travail selon le secteur

\begin{tabular}{|l|c|c|c|c|c|}
\hline & $\begin{array}{c}\text { Trois fonctions } \\
\text { publiques }\end{array}$ & Dont administration & Dont éducation & Dont santé & $\begin{array}{c}\text { Ensemble } \\
\text { des entreprises }\end{array}$ \\
\hline 1991 & 30 & 32 & 31 & 19 & 33 \\
\hline 1998 & 53 & 60 & 55 & 36 & 49 \\
\hline 2005 & 66 & 67 & 71 & 56 & 58 \\
\hline
\end{tabular}

Sources : enquêtes complémentaires aux enquêtes emploi sur les conditions de travail, Insee et Dares. 
secrétariat, saisie, etc.) associées à une activité principale de type industriel par exemple. La distribution de l'accès à l'informatique selon l'âge dans le privé et le public pourrait ainsi d'abord résulter des activités effectuées : les salariés âgés du privé pourraient moins utiliser l'informatique simplement parce qu'ils seraient prioritairement affectés à des tâches non informatisées (4), plus ou moins répandues selon le secteur.

Une information présente dans l'enquête $C O I$ 2006 permet de tenir compte - au moins en partie - de la particularité de ces activités visà-vis de l'utilisation des outils informatiques. L'ensemble des salariés, y compris les agents de l'État, décrivent leur activité à travers des fonctions génériques communes : exploitation-production, comptabilité-gestion, secrétariat-accueil-saisie, par exemple. Ces fonctions sont en effet très diversement informatisées (cf. tableau 1), et le taux d'utilisation de l'informatique par les salariés exerçant ces fonctions est relativement proche d'un secteur à l'autre - à l'exception des fonctions hétérogènes comme la « Manutention, magasinage, logistique » ou la fonction « Autres ».

Par ailleurs, les catégories socioprofessionnelles auxquelles appartiennent les salariés déterminent également le degré d'équipement et les types d'utilisation de l'informatique. Tenir compte des différents secteurs d'activité du privé (services aux entreprises, activités financières ou industrie, construction etc.) et de la répartition de la main-d'œuvre selon les fonctions et les catégories socioprofessionnelles permet donc de neutraliser partiellement la diversité des tâches des salariés du privé et des agents de la fonction publique de l'État, diversité qui peut expliquer une grande partie des différences dans l'utilisation de l'informatique. Il demeure néanmoins une hétérogénéité inobservée des activités, dont nos conclusions tiennent compte.

L'échelle d'observation de l'employeur pose un problème épineux : on ne peut pas faire une analogie stricte entre les directions (centrales ou déconcentrées) de ministères et les entreprises, car si l'organisation s'observe au niveau local, la gestion de la main-d'œuvre répond à des normes et règlements nationaux. Doit-on comparer la fonction publique de l'État à une très grande entreprise ou à un groupe contrôlant des entreprises de diverses tailles ? Par ailleurs, l'équipement en outils informatiques, particulièrement les plus élaborés, est étroitement lié à la taille des entreprises et à leur appartenance à un groupe (Besnard et al., 2007). Pour les comparer aux directions de la fonction publique de l'État, nous avons pris en compte dans un premier temps à la fois la taille des entreprises et leur appartenance à un groupe. L'utilisation de l'une et/ou l'autre de ces caractéristiques ne modifie guère nos résultats : nous avons finalement choisi de comparer les directions de la fonc-

4. Comme, par exemple, les activités de type relationnel, où l'âge ou l'expérience peuvent représenter un avantage comparatif.

Tableau 1

Taux d'équipement informatique par fonction et par secteur

\begin{tabular}{|c|c|c|c|c|c|c|}
\hline & Industrie & $\begin{array}{l}\text { Construction- } \\
\text { transport }\end{array}$ & Commerce & $\begin{array}{c}\text { Services aux } \\
\text { entreprises }\end{array}$ & $\begin{array}{c}\text { Activités } \\
\text { financières }\end{array}$ & $\begin{array}{l}\text { Fonction } \\
\text { publique } \\
\text { de l'État }\end{array}$ \\
\hline Production, chantiers, exploitation & 58 & 29 & 61 & 53 & 71 & 61 \\
\hline Installation, réparation, maintenance & 79 & 45 & 79 & 85 & 83 & 62 \\
\hline Nettoyage, gardiennage & 13 & 19 & 14 & 17 & 28 & 11 \\
\hline Manutention, magasinage, logistique & 69 & 37 & 66 & 65 & 23 & 76 \\
\hline Gestion, comptabilité & 99 & 100 & 95 & 97 & 100 & 100 \\
\hline Commercial, secrétariat, accueil & 95 & 95 & 89 & 95 & 90 & 93 \\
\hline Études, recherche et développement & 99 & 98 & 99 & 99 & 100 & 97 \\
\hline Autre fonction & 92 & 52 & 75 & 81 & 77 & 96 \\
\hline
\end{tabular}

Lecture : $61 \%$ des agents de la fonction publique de l'État exerçant une fonction de production, chantier ou exploitation utilisent au moins un équipement informatique au travail. C'est le cas de la totalité des agents exerçant une fonction gestion ou comptabilité.

Champ : fonction publique de l'Etat hors enseignants, magistrats, agents du ministère de la Défense et des établissements publics. Pour les secteurs marchands : salariés des entreprises marchandes non agricoles de plus de 20 salariés.

Sources : COI 2006, Statistique publique, DGAFP-Dares-CEE. 
tion publique de l'État aux entreprises appartenant à un groupe. Pour analyser les conditions d'accès aux outils selon les caractéristiques des salariés au sein d'organisations ayant des niveaux d'équipement technologique comparables, nous avons également pris en compte des indicateurs de ce niveau d'équipement issus du volet « employeurs » de l'enquête : l'existence d'un progiciel de gestion intégré (ERP, pour Enterprise Resource Planning (5)) et d'outils de travail collaboratif au sein de l'organisation («groupware » (6), visio-conférence...).

\section{Des agents de l'État plus âgés et plus anciens et une utilisation intensive de l'informatique}

Compte tenu de l'historique des recrutements et du maintien dans l'emploi de ses agents (Gautier et al., 2009), la fonction publique de l'État est l'un des secteurs dans lequel la proportion de seniors est la plus importante, et cette part s'accroît régulièrement. La proportion d'agents titulaires des ministères âgés de 50 ans et plus atteignait $28 \%$ en 2005 et $32 \%$ en 2007 , contre respectivement $21 \%$ et $24 \%$ parmi les salariés du privé (Baëhr, 2008 ; DGAFP, 2009). Plus âgés, les agents de l'État ont aussi une plus grande ancienneté que les salariés du privé : selon l'enquête COI (7), un peu plus du quart de ces derniers travaillent dans la même entreprise depuis au moins 18 ans, mais près de la moitié des agents de l'État sont dans le même cas (cf. graphique I).
Les agents de la fonction publique de l'État sont davantage équipés d'outils informatiques que les salariés de l'ensemble des secteurs marchands (cf. tableau 2), et sont particulièrement nombreux à utiliser Internet, un intranet, ou à alimenter des bases de données. Leur usage des outils informatiques est comparable à celui des salariés des banques et assurances. Mais, comme on l'a vu, l'utilisation de l'informatique est très liée aux fonctions exercées. Lorsque l'on prend en compte le secteur, la fonction et la PCS, l'équipement des agents de l'État en

\section{Graphique I \\ Distribution des salariés par année d'embauche et par secteur}

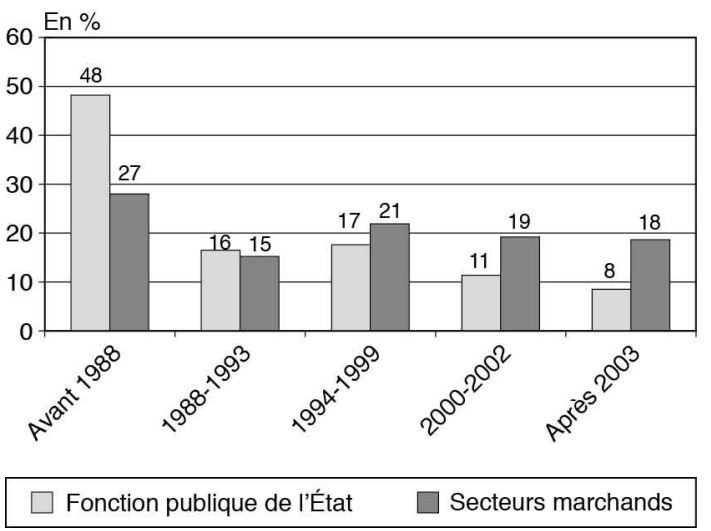

Lecture : sur le champ COI, $48 \%$ des agents de la fonction publique de l'État ont été embauchés avant 1988, contre $27 \%$ des salariés de l'ensemble des secteurs marchands.

Champ : fonction publique de l'État hors enseignants, magistrats, agents du ministère de la Défense et des établissements publics. Pour les secteurs marchands : salariés des entreprises marchandes non agricoles de plus de 20 salariés.

Source : COI 2006, statistique publique, DGAFP-Dares-CEE.

Tableau 2

Équipements et utilisation de l'informatique selon le secteur Salariés du privé appartenant à un groupe et agents de la fonction publique

\begin{tabular}{|c|c|c|c|c|c|c|}
\hline & Industrie & $\begin{array}{l}\text { Construction- } \\
\text { transport }\end{array}$ & Commerce & $\begin{array}{l}\text { Services aux } \\
\text { entreprises }\end{array}$ & $\begin{array}{c}\text { Activités } \\
\text { financières }\end{array}$ & $\begin{array}{l}\text { Fonction } \\
\text { publique } \\
\text { de l'État }\end{array}$ \\
\hline Formulaires électroniques & 34 & 20 & 25 & 40 & 62 & 44 \\
\hline Accès à Internet & 37 & 34 & 37 & 52 & 53 & 57 \\
\hline Outils électroniques collaboratifs & 45 & 34 & 40 & 52 & 67 & 55 \\
\hline Mél individuel & 50 & 35 & 37 & 56 & 77 & 67 \\
\hline Bases de données en ligne & 48 & 35 & 43 & 52 & 76 & 71 \\
\hline Utilisation de l'intranet & 54 & 37 & 48 & 57 & 79 & 75 \\
\hline Accès restreint à Internet & 13 & 13 & 13 & 16 & 24 & 28 \\
\hline Accès non restreint à Internet & 24 & 21 & 24 & 36 & 29 & 30 \\
\hline
\end{tabular}

Lecture : $20 \%$ des salariés du secteur construction-transports et $44 \%$ des agents de la fonction publique de l'État du champ de l'enquête COI 2006 utilisent l'informatique pour remplir des formulaires électroniques.

Champ : fonction publique de l'État hors enseignants, magistrats, agents du ministère de la Défense et des établissements publics. Pour les secteurs marchands : salariés des entreprises marchandes non agricoles de plus de 20 salariés appartenant à un groupe. Sources : COI 2006, statistique publique, DGAFP-Dares-CEE. 
ordinateurs est ainsi comparable à celui des salariés des entreprises appartenant à un groupe (Guillemot et Peyrin, 2009).

\section{L'accès à l'informatique des agents de la fonction publique de l'État ne diminue pas avec l'âge}

D ans l'ensemble des secteurs marchands, $77 \%$ des trentenaires utilisent au moins un équipement informatique ; cette proportion décroît ensuite jusqu'à $68 \%$ après 50 ans. Dans la fonction publique de l'État, elle dépasse $90 \%$ avant 30 ans (8), redescend jusqu'à $83 \%$ pour les $40-49$ ans, puis remonte à $87 \%$ après 49 ans (cf. graphique II).

Si une majorité des seniors en poste a aujourd'hui accès à l'informatique, la proportion des nonutilisateurs après 50 ans reste élevée dans les secteurs marchands. Comme dans les années 1990, ces non-utilisateurs sont essentiellement des ouvriers et des salariés ayant un faible niveau de formation initiale. Pour autant, le contrôle de ces variables dans un modèle Logit (cf. encadré 2) ne supprime pas un effet négatif de l'âge sur la probabilité d'avoir accès à l'informatique au travail après 50 ans (cf. tableau 3 , modèle $1 \mathrm{Ma}$ ). La moindre utilisation de l'informatique après 50 ans persiste donc dans le

\section{Graphique II \\ Part des salariés utilisant au moins un équipement informatique, par secteur et par âge}

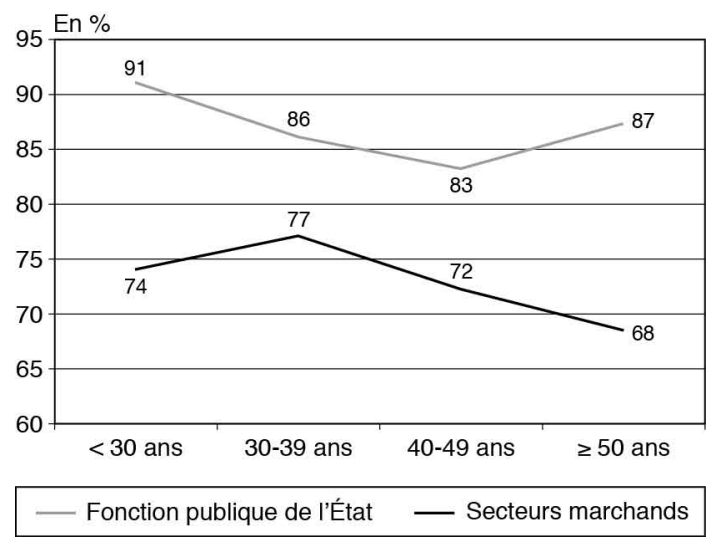

Lecture : $91 \%$ des agents de la fonction publique de l'État âgés de moins de 30 ans et $87 \%$ de ceux âgés de 50 ans et plus utilisent au moins un équipement informatique.

Champ : fonction publique de l'État hors enseignants, magistrats, agents du ministère de la Défense et des établissements publics. Pour les secteurs marchands : salariés des entreprises marchandes non agricoles de plus de 20 salariés.

Source : COI 2006, statistique publique, DGAFP-Dares-CEE. privé. En revanche, le même modèle appliqué aux agents de l'État montre que leur probabilité d'accès à l'ordinateur ne varie quasiment pas avec l'âge (cf. tableau 4, modèle $1 \mathrm{~Pa}$ ). Il s'agit là du premier indice d'un résultat peu conforme à l'idée qu'après 50 ans, les salariés seraient intrinsèquement moins "capables » d'utiliser un ordinateur. L'introduction dans le modèle de l'ancienneté en plus de l'âge, ainsi que d'un indicateur d'entraide (cf. infra) (cf. tableaux 3 et 4 , modèles $1 \mathrm{Mb}$ et $1 \mathrm{~Pb}$ ), fait apparaître qu'une plus grande ancienneté augmente la probabilité d'accès à l'informatique quel que soit le secteur. Les agents de l'État âgés de cinquante ans et plus, dont l'ancienneté est d'au moins quinze ans (9), ont ainsi un accès à l'informatique non significativement différent de celui de leurs collègues plus jeunes (toutes anciennetés confondues), mais supérieur à celui des quinquagénaires ayant une ancienneté plus faible : l'ancienneté compenserait ainsi l'effet de l'âge.

\section{L'ancienneté a un effet rôle positif dans l'accès à l'informatique}

L'ancienneté chez l'employeur conditionne l'accumulation d'un capital humain spécifique permettant de maîtriser les outils informatiques, notamment par le biais des formations formelles ou informelles. L'acquisition de compétences en informatique après la fin de la formation initiale, et donc essentiellement via la formation professionnelle, peut cependant être liée à l'ancienneté sur le marché du travail plutôt qu'à l'ancienneté chez le même employeur. Les connaissances en informatique de gestion sont par exemple généralement transférables d'une entreprise à l'autre. Si les deux types d'ancien-

\footnotetext{
5. Un progiciel de gestion intégrée, ou ERP, est un ensemble de logiciels qui, s'appuyant sur une base de données centrale, permet de gérer l'ensemble des processus opérationnels d'une ou plusieurs des fonctions d'une entreprise comme la gestion des ressources humaines ou la gestion comptable et financière. 6. Logiciels qui permettent à des utilisateurs séparés géographiquement de travailler ensemble en étant reliés par un réseau informatique. Cela peut passer par le partage des messageries, des agendas, des bases de documents, etc. Outlook et Lotus Notes ont par exemple des fonctionnalités de groupware.

7. L'enquête $\mathrm{COI}$ interroge une cohorte de salariés présents dans l'entreprise depuis au moins 10 mois : l'ancienneté mesurée par $\mathrm{COI}$ est donc plus importante que celle qui serait mesurée par un échantillon représentatif de salariés présent au moment de l'entretien, comme dans l'enquête emploi de l'Insee, par exemple. Compte tenu de cette caractéristique de l'échantillon, on y compte très peu d'agents non titulaires parmi les salariés de l'État: dans tout l'article, nous considérons que l'analyse porte sur les agents de l'État (champ COI) titulaires, bénéficiant de la garantie de l'emploi.

8. Mais le nombre d'agents de la fonction publique de moins de 30 ans est faible dans l'échantillon.

9. Ces résultats sont robustes à différentes spécifications de l'ancienneté, voir encadré 2.
} 
neté se confondent souvent dans la fonction publique de l'État, c'est nettement moins le cas dans les secteurs marchands où la mobilité interentreprises est plus répandue. Dans ce cas, l'ancienneté dans l'entreprise sous-estime la maîtrise des outils informatiques acquise sur le marché du travail, et l'âge capte probablement une partie de celle-ci. Les effets positifs

\section{Encadré 2}

\section{MODÉLISATION DE L'UTILISATION DE L'INFORMATIQUE}

Des modèles logistiques distincts ont été réalisés pour la fonction publique de l'État d'une part, et l'ensemble des secteurs marchands d'autre part. L'échantillon de la fonction publique de l'État comprend 955 observations, celui des entreprises marchandes 14369 ; ces différences de taille d'échantillon pourraient expliquer que des résultats soient non significatifs dans un cas, et significatifs dans l'autre. Les modèles ont donc été testés pour différents secteurs marchands, avec des échantillons de taille similaire à celui de la fonction publique de l'État : sur la variable qui nous intéresse, c'est-à-dire l'âge, les résultats sont qualitativement les mêmes (l'effet de l'âge apparaît notamment dans le modèle 1a), sauf dans le cas notoire des banques et assurances. Dans ce secteur, les résultats sont, dans l'ensemble, très proches de ceux de la fonction publique de l'État, alors que ce n'est pas le cas, par exemple, des services aux entreprises - même si on exclut les services de nettoyage et autres services où l'utilisation de l'informatique est faible. La plupart des banques et assurances (tout au moins les plus grandes d'entre elles) fonctionnent également selon un modèle de marché interne et, quel que soit leur âge, la quasitotalité des salariés y disposent d'un ordinateur - ce qui peut expliquer la proximité des résultats avec ceux de la fonction publique de l'État.

Nous avons dans un premier temps modélisé un score de variété d'utilisation de l'informatique (cf. annexe 1), pouvant prendre neuf positions ordonnées (regroupées en quatre modalités), ce qui incite à utiliser une modélisation logistique polytomique ordonnée. Mais l'hypothèse de proportionnalité des pentes (hypothèse d'égalité des coefficients de la régression de la modalité la plus basse à la modalité la plus haute) n'est pas vérifiée. Finalement, plusieurs modélisations logistiques dichotomiques permettent de vérifier la robustesse des résultats selon différents seuils de la variable de score, et donc différents usages de l'informatique : les modèles $1 \mathrm{M}$ et $1 \mathrm{P}$ présentent, respectivement pour les secteurs marchands $(M)$ et la fonction publique de l'État $(P)$, la probabilité d'utilisation de l'informatique (quel qu'en soit le niveau d'utilisation, de basique à variée) versus l'absence d'utilisation (cf. tableaux 3 et 4) ; les modèles 2M (cf. tableau 5) et 2P (cf. tableau 6) une utilisation intermédiaire ou variée versus l'absence d'utilisation ou une utilisation basique ; les modèles $3 \mathrm{M}$ (cf. tableau 7) et 3P (cf. tableau 8) une utilisation variée versus la non utilisation, une utilisation basique ou intermédiaire.

Les variables exogènes ont été introduites en plusieurs étapes, pour distinguer l'effet des variables de contrôle de celui des variables d'intérêt. Les variables de contrôle décrivent les caractéristiques du poste de travail : la fonction (production-manutention-nettoyage, secrétariat-saisie-accueil, comptabilité-gestion, études-recherche et développementenseignement-soin, autres fonctions), la catégorie socioprofessionnelle (ouvriers, employés, professions intermédiaires, cadres) et les caractéristiques du salarié : le sexe, le diplôme (inférieur au bac, supérieur au bac, $2^{\mathrm{e}}$ cycle universitaire). S'ajoutent à ces variables usuelles deux éléments caractéristiques de l'employeur : le fait d'appartenir à un groupe (dans le cas des secteurs marchands) et le niveau d'équipement en outils TIC au niveau de l'entreprise ou de l'administration. Deux variables ont été retenues comme indicatrices de l'équipement informatique de l'entreprise : la mise en place d'un progiciel de gestion intégré (ERP) et d'outils de travail collaboratif. Les ERP structurent fortement les organisations par la standardisation et la centralisation des circuits d'information ; les outils de travail collaboratif équipent le travail à distance.

Les variables d'intérêt sont l'âge, l'ancienneté, et l'entraide (pour la mesure de l'entraide, voir annexe 2). Les tableaux 3 et 4 présentent dans le cas du premier modèle (probabilité d'utilisation de l'informatique quel que soit le niveau versus l'absence d'utilisation), les résultats avant introduction de l'ancienneté et de l'entraide (cf. modèles $1 \mathrm{Ma}$ et $1 \mathrm{~Pa}$ ), puis les résultats après l'introduction de ces variables (modèles $1 \mathrm{Mb}$ et $1 \mathrm{~Pb}$ ). Dans le cas des modèles 2 et 3 , la même démarche d'introduction progressive des variables a été adoptée. Les résultats sont qualitativement robustes aux différentes spécifications d'ancienneté et d'âge testées (nombre d'années d'ancienneté et âge séparés, tranches d'ancienneté croisées avec l'âge en faisant varier les seuils...). Nous avons finalement choisi d'introduire l'ancienneté (nombre d'années de présence dans l'entreprise ou l'administration) croisée avec l'âge, avec un même seuil de 15 ans pour les trois tranches d'âge (les moins de 30 ans étant alors regroupés avec les 30-39 ans). On retrouve des résultats proches lorsque l'on compare les salariés de plus ou moins 10, 20 ou 25 ans d'ancienneté - ces deux derniers seuils sont plus réalistes pour les quinquagénaires de la fonction publique, mais ils impliquent de regrouper les autres tranches d'âge. Par ailleurs, le nombre d'années d'ancienneté, introduit de façon séparée de l'âge, apparaît significativement favorable à l'utilisation de l'informatique dans tous les secteurs. Dans la fonction publique de l'État, l'introduction de l'ancienneté fait apparaître un tel effet défavorable de l'âge, qui était masqué lorsqu'on ne tient pas compte de cette variable : dans le public, l'ancienneté, qui élève la probabilité d'utilisation de l'informatique, compense en quelque sorte l'âge. 
de l'ancienneté et négatifs de l'âge sur l'accès à l'informatique seraient alors sous-estimés, et ce davantage dans les secteurs marchands que dans la fonction publique de l'État.

L'ancienneté dans l'entreprise peut également traduire des effets de sélection : les salariés anciens sont les « survivants » d'un processus de sélection qui peut prendre en compte, parmi d'autres dimensions, la capacité (inobservée) à maîtriser les nouvelles technologies. Cette sélection, probablement présente dans nombre d'entreprises marchandes, ne concerne pas les agents de la fonction publique de l'État, où

Tableau 3

Modélisation de l'utilisation de l'informatique : utilisation d'un ordinateur (tous niveaux) Secteurs marchands

\begin{tabular}{|c|c|c|c|}
\hline & & \multicolumn{2}{|c|}{$\begin{array}{l}\text { Utilisation d'un ordinateur (tous niveaux) } \\
\text { versus pas d'utilisation }\end{array}$} \\
\hline & & MODELE $1 \mathrm{Ma}$ & MODELE $1 \mathrm{Mb}$ \\
\hline Constante & & $1,10^{\star \star \star}(0,117)$ & $0,85^{* \star *}(0,131)$ \\
\hline \multirow[t]{2}{*}{ Secteur d'activité de l'entreprise } & Commerce & $-0,28^{\star * \star}(0,075)$ & $-0,26$ *** $(0,076)$ \\
\hline & Construction-transport & $-0,92^{\star \star \star}(0,074)$ & $-0,85^{\star \star \star}(0,075)$ \\
\hline \multirow[t]{2}{*}{ Réf. : industrie } & Finances & $-0,51^{\star \star \star}(0,116)$ & $-0,42$ *** $(0,119)$ \\
\hline & Services aux entreprises & $-0,75^{\star * \star}(0,087)$ & $-0,59$ *** $(0,090)$ \\
\hline \multicolumn{4}{|l|}{ L'entreprise appartient à un groupe } \\
\hline Réf. : non & Groupe & $0,55^{* \star *}(0,058)$ & $0,50^{* * *}(0,059)$ \\
\hline \multicolumn{4}{|l|}{ L'entreprise est équipée en ERP } \\
\hline Réf. : non & ERP & $0,22^{* * \star}(0,055)$ & $0,18^{* \star \star}(0,055)$ \\
\hline \multicolumn{4}{|l|}{$\begin{array}{l}\text { L'entreprise est équipée en outils } \\
\text { de travail collaboratif }\end{array}$} \\
\hline Réf. : non & Travail collaboratif & $0,64^{* \star *}(0,056)$ & $0,58^{* * *}(0,057)$ \\
\hline \multirow[t]{2}{*}{ Fonction du salarié } & Production, nettoyage, manutention & $-1,53^{\star \star \star}(0.101)$ & $-1,53$ *** $(0,102)$ \\
\hline & Comptabilité, gestion & $2,11^{\star \star \star}(0,315)$ & $2,13^{\star \star \star}(0,316)$ \\
\hline \multirow[t]{2}{*}{ Réf. : secrétariat, saisie, commercial } & Études, R\&D, enseignement, soin & $-0,43^{\star \star}(0,188)$ & $-0,38^{\star \star}(0,190)$ \\
\hline & Autres fonctions & $-1,11^{\star \star \star}(0,118)$ & $-1,05^{\star \star \star}(0,119)$ \\
\hline PCS & Cadres & 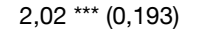 & $1,94^{\star \star \star}(0,194)$ \\
\hline \multirow[t]{2}{*}{ Réf. : employé } & Professions intermédiaires & 0,62 *** $(0,096)$ & $0,57^{\star \star \star}(0,097)$ \\
\hline & Ouvriers & $-1,18^{\star * \star}(0,091)$ & $-1,14^{\star \star *}(0,092)$ \\
\hline \multicolumn{4}{|l|}{ Sexe } \\
\hline Réf. : femme & Homme & $0,23^{\star \star \star}(0,060)$ & $0,19^{\star \star \star}(0,061)$ \\
\hline Diplôme & Supérieur ou égal au bac & 1,38 *** $(0,092)$ & $1,36^{\star \star \star}(0,093)$ \\
\hline Réf. : primaire ou technique court & Secondaire & 0,74 *** $(0,069)$ & $0,74^{\star \star \star}(0,069)$ \\
\hline \multirow[t]{2}{*}{ Âge } & Moins de 30 ans & $0,35^{* \star \star}(0,085)$ & \\
\hline & $30-39$ ans & $0,49^{* \star *}(0,072)$ & \\
\hline Réf. : 50 ans et plus & $40-49$ ans & $0,32 * * *(0,069)$ & \\
\hline \multirow[t]{3}{*}{ Âge et ancienneté } & Moins de 40 ans, ancienneté $<15$ ans & & $0,16 *(0,086)$ \\
\hline & Moins de 40 ans, ancienneté $>=15$ ans & & $0,43^{\star * \star}(0,122)$ \\
\hline & 40-49 ans, ancienneté< 15 ans & & - 0,04 n.s. $(0,099)$ \\
\hline \multirow{2}{*}{$\begin{array}{l}\text { Réf. : } 50 \text { ans et plus, ancienneté } \\
\text { supérieure à } 15 \text { ans }\end{array}$} & $40-49$ ans, ancienneté $>=15$ ans & & $0,29 * \star *(0,093)$ \\
\hline & $>=50$ ans, ancienneté $<15$ ans & & $-0,32^{\star \star \star}(0,111)$ \\
\hline Score d'entraide & Moyenne & & $0,62 * \star *(0,058)$ \\
\hline Réf. : entraide basse & Élevée & & $0,88^{* * *}(0,068)$ \\
\hline
\end{tabular}

Lecture : le modèle 1 Ma présente la probabilité d'utilisation de l'informatique (quel qu'en soit le niveau d'utilisation, de basique à varié) versus l'absence d'utilisation dans les secteurs marchands (voir encadré 2), avant introduction de l'ancienneté et de l'entraide ; $1 \mathrm{Mb}$ après l'introduction de ces variables. La situation de référence est signalée dans la première colonne. Les coefficients sont issus de l'estimation des modèles Logit. Les écarts-types sont présentés entre parenthèses.

Dans les secteurs marchands, la probabilité d'utiliser un ordinateur (plutôt que de ne pas l'utiliser) d'un salarié âgé de moins de 30 ans est significativement plus élevée que celle d'un salarié de 50 ans et plus.

n.s. non significatif ; ${ }^{* *}$ significatif au seuil de $1 \%$, ** significatif au seuil de $5 \%,{ }^{*}$ significatif au seuil de $10 \%$.

Champ : salariés des entreprises marchandes non agricoles de plus de 20 salariés.

Sources : fichier couplé dans la fonction publique de l'État, enquête COI couplée 2006, statistique publique, Insee-Dares-CEE. 
l'ancienneté est cependant favorable à l'utilisation de l'informatique. Enfin, une autre interprétation a été avancée dans les années 1990 : les salariés les plus anciens, bénéficiant de la confiance de leur employeur, se voyaient plus facilement confier un outil coûteux et puissant (Cézard et al., 2000). L'ordinateur s'est depuis banalisé et son prix a considérablement baissé, mais l'accès à ces outils reste peut-être encore une question de confiance, dans la mesure où ils permettent d'accéder à des informations sur l'entreprise, ou parce qu'ils peuvent, s'agissant d'ordinateurs reliés à l'Internet, être détournés pour des usages extraprofessionnels.

\section{Dans la fonction publique de l'État, les plus de 50 ans ont une utilisation variée des outils informatiques}

La plupart des agents du public (et des salariés des services marchands) utilisent aujourd'hui un ordinateur. Le simple accès aux outils ne serait donc plus susceptible de les discriminer selon leur âge. Mais les salariés vieillissants

Tableau 4

Modélisation de l'utilisation de l'informatique : utilisation d'un ordinateur (tous niveaux)

Fonction publique de l'État

\begin{tabular}{|c|c|c|c|}
\hline & & \multicolumn{2}{|c|}{$\begin{array}{l}\text { Utilisation d'un ordinateur (tous niveaux) } \\
\text { versus pas d'utilisation }\end{array}$} \\
\hline & & MODELE $1 \mathrm{~Pa}$ & MODELE 1Pb \\
\hline Constante & & $2,07^{\star \star \star}(0,465)$ & $1,85^{* \star \star}(0,570)$ \\
\hline $\begin{array}{l}\text { L'administration est équipée en ERP } \\
\text { Réf. : non }\end{array}$ & ERP & 0,27 n.s. $(0,280)$ & 0,25 n.s. $(0,289)$ \\
\hline $\begin{array}{l}\text { L'administration est équipée en } \\
\text { outils de travail collaboratif } \\
\text { Réf. : non }\end{array}$ & Travail collaboratif & - 0,11 n.s. $(0,295)$ & - 0,02 n.s. $(0,309)$ \\
\hline $\begin{array}{l}\text { Fonction du salarié } \\
\text { Réf. : secrétariat, saisie, commercial }\end{array}$ & $\begin{array}{l}\text { Production, nettoyage, manutention } \\
\text { Etudes, R\&D, enseignement, soin } \\
\text { Autres fonctions }\end{array}$ & $\begin{array}{l}-4,34^{* * *}(0,488) \\
-1,72^{\star * *}(0,609) \\
-1,30^{*}(0,534)\end{array}$ & $\begin{array}{l}-4,45^{* \star \star}(0,546) \\
-1,44^{\star *}(0,637) \\
-1,45^{\star \star \star}(0,546)\end{array}$ \\
\hline $\begin{array}{l}\text { PCS } \\
\text { Réf. : employé }\end{array}$ & $\begin{array}{l}\text { Cadres } \\
\text { Professions intermédiaires } \\
\text { Ouvriers }\end{array}$ & $\begin{array}{c}1,83^{*}(0,733) \\
1,83^{* * *}(0,475) \\
0,41 \text { n.s. }(0,362)\end{array}$ & $\begin{array}{c}1,57^{* *}(0,748) \\
1,98^{* \star *}(0,508) \\
0,31 \text { n.s. }(0,383)\end{array}$ \\
\hline $\begin{array}{l}\text { Sexe } \\
\text { Réf. : femme }\end{array}$ & Homme & $1,05^{\star \star \star}(0,320)$ & 0,80 ** $(0,333)$ \\
\hline $\begin{array}{l}\text { Diplôme } \\
\text { Réf. : primaire ou technique court }\end{array}$ & $\begin{array}{l}\text { Supérieur ou égal au bac } \\
\text { Secondaire }\end{array}$ & $\begin{array}{l}1,35^{* \star *}(0,473) \\
1,22^{\star * *}(0,356) \\
\end{array}$ & $\begin{array}{l}1,24^{\star \star \star}(0,495) \\
1,10^{\star \star \star}(0,368) \\
\end{array}$ \\
\hline $\begin{array}{l}\text { Âge } \\
\text { Réf. : } 50 \text { ans et plus }\end{array}$ & $\begin{array}{l}\text { Moins de } 30 \text { ans } \\
30-39 \text { ans } \\
40-49 \text { ans }\end{array}$ & $\begin{array}{c}-0,05 \text { n.s. }(0,781) \\
0,39 \text { n.s. }(0,389) \\
0,57^{*}(0,324)\end{array}$ & \\
\hline $\begin{array}{l}\text { Âge et ancienneté } \\
\text { Réf. : } 50 \text { ans et plus, ancienneté supé- } \\
\text { rieure à } 15 \text { ans }\end{array}$ & $\begin{array}{l}\text { Moins de } 40 \text { ans, ancienneté }<15 \text { ans } \\
\text { Moins de } 40 \text { ans, ancienneté }>=15 \text { ans } \\
40-49 \text { ans, ancienneté }<15 \text { ans } \\
40-49 \text { ans, ancienneté }>=15 \text { ans } \\
>=50 \text { ans, ancienneté }<15 \text { ans }\end{array}$ & & $\begin{array}{l}-0.03 \text { n.s. }(0,463) \\
-0,69 \text { n.s. }(0,759) \\
-0.13 \text { n.s. }(0,493) \\
0.33 \text { n.s. }(0,432) \\
-1.04 \text { ** }(0,479)\end{array}$ \\
\hline $\begin{array}{l}\text { Score d'entraide } \\
\text { Réf. : entraide basse }\end{array}$ & $\begin{array}{l}\text { Moyenne } \\
\text { Élevée }\end{array}$ & & $\begin{array}{l}0,77^{* *}(0,332) \\
1,52^{* * \star}(0,423)\end{array}$ \\
\hline
\end{tabular}

Lecture : le modèle 1Pa présente la probabilité d'utilisation de l'informatique (quel qu'en soit le niveau d'utilisation, de basique à varié) versus l'absence d'utilisation dans la fonction publique de l'État (voir encadré 2), avant introduction de l'ancienneté et de l'entraide ; $1 \mathrm{~Pb}$ après l'introduction de ces variables. La situation de référence est signalée dans la première colonne. Les coefficients sont issus de l'estimation des modèles Logit. Les écarts-types sont présentés entre parenthèses.

Dans la fonction publique de l'Etat, la probabilité d'utiliser un ordinateur (plutôt que de ne pas l'utiliser) d'un agent de moins de 30 ans n'est pas significativement différente de celle d'un agent de 50 ans et plus.

n.s. non significatif ; *** significatif au seuil de $1 \%$, ${ }^{* *}$ significatif au seuil de $5 \%$, * significatif au seuil de $10 \%$.

Champ : fonction publique de l'État, hors enseignants, magistrats, agents du ministère de la Défense et des établissements publics, et hors fonction "gestion-comptabilité ". Dans la fonction publique, cette fonction est en effet complètement équipée en ordinateurs : les observations correspondantes ont été supprimées du modèle.

Sources : fichier couplé dans la fonction publique de l'État, enquête COI couplée 2006, statistique publique, DGAFP - CEE. 
ont-ils autant accès que les autres à des outils plus récents, autorisant des usages plus intenses, variés ou plus avancés ? Cette question a été posée dès le début de la diffusion massive de ces outils, tant ils sont marqués depuis l'origine par des changements permanents : «La rapidité des changements qui semble désormais caractériser la technologie risque, en effet, d'introduire entre des phases successives de l'informatique autant de distance qu'entre les systèmes de travail traditionnels et informatisés, et perpétuer ainsi le problème [d'exclusion des plus âgés] au-delà des générations actuelles 》 (Marquié et Baracat, 1992). Un score d'utilisation plus ou moins avancée de l'informatique, construit à partir de cinq questions posées dans $C O I$ sur l'utilisation professionnelle de l'Internet, de la messagerie électronique, d'un intranet, et la connexion à distance au système d'information de l'entreprise (cf. annexe 1) permet d'apporter des éléments de réponse à cette question. Le score distingue trois niveaux d'utilisation de l'informatique : un niveau basique (utilisation d'un ordinateur, sans aucune des fonctionnalités prise en compte - soit essentiellement pour faire de la saisie), un niveau intermédiaire et un niveau d'utilisation variée, cumulant la plupart de ces fonctionnalités.

La fonction publique de l'État se distingue par une proportion élevée d'utilisateurs d'informatique de niveau intermédiaire (plus de la moitié) alors que les salariés de l'ensemble des secteurs

\section{Graphique III \\ Distribution du score d'utilisation \\ de l'informatique par secteur}

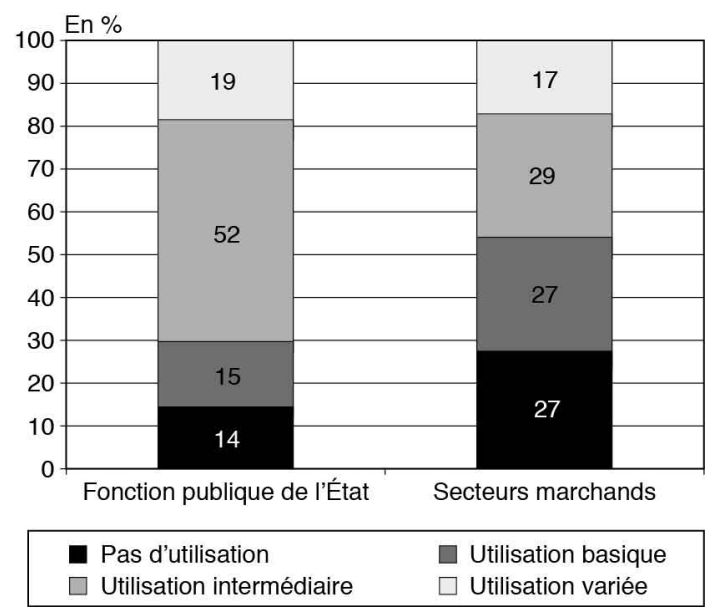

Lecture : $19 \%$ des agents de la fonction publique de l'État et $17 \%$ des salariés des secteurs marchands ont une utilisation variée de l'informatique.

Champ : fonction publique de l'État hors enseignants, magistrats, agents du ministère de la Défense et des établissements publics. Pour les secteurs marchands : salariés des entreprises marchandes non agricoles de plus de 20 salariés.

Source : COI 2006, statistique publique, DGAFP-Dares-CEE. marchands se distribuent à un peu près également entre non-utilisateurs, utilisateurs basiques et intermédiaires, l'utilisation variée étant moins fréquente (cf. graphique III). Par ailleurs, l'utilisation d'outils informatiques avancés augmente avec l'âge dans la fonction publique de l'État, mais pas dans les autres secteurs (cf. graphique IV).

\section{Graphique IV \\ A - Distribution du score d'utilisation de l'informatique selon l'âge - \\ Secteurs marchands}

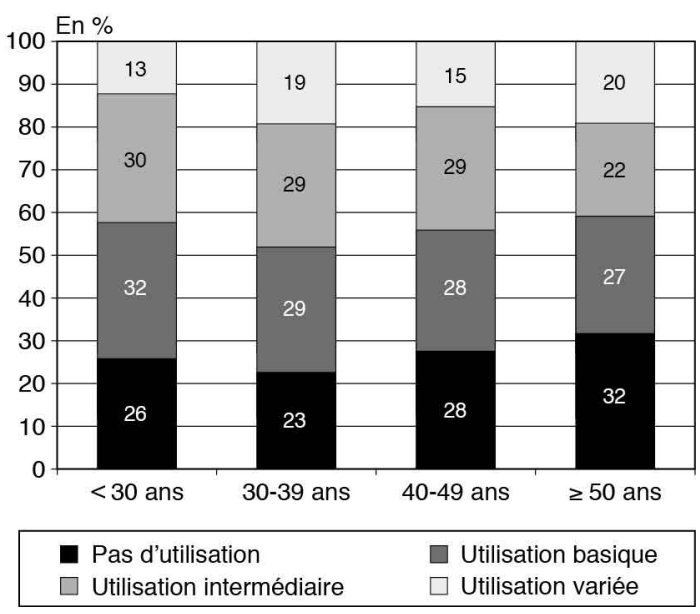

Lecture : dans l'ensemble des secteurs marchands, la part des utilisateurs de niveau varié est de $13 \%$ avant 30 ans, $19 \%$ entre 30 et 39 ans, $15 \%$ entre 40 et 49 ans et $20 \%$ à partir de 50 ans.

Champ : salariés des entreprises marchandes non agricoles de plus de 20 salariés.

Source : COI 2006, statistique publique, DGAFP-Dares-CEE.

\section{B - Distribution du score d'utilisation de l'informatique selon l'âge - Fonction publique de l'État}

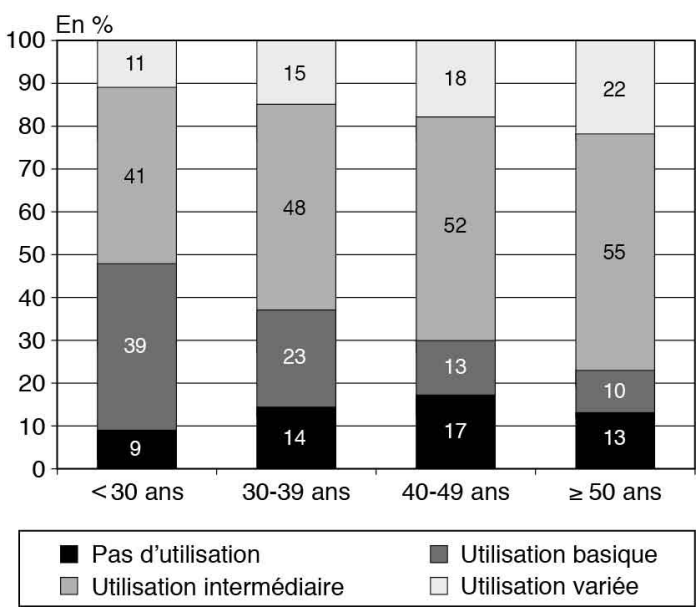

Lecture : dans la fonction publique d'État, la part des utilisateurs de niveau varié augmente avec l'âge : elle est de $11 \%$ avant 30 ans, de $15 \%$ entre 30 et 39 ans, $18 \%$ entre 40 et 49 ans et $22 \%$ à partir de 50 ans.

Champ : fonction publique de l'État hors enseignants, magistrats, agents du ministère de la Défense et des établissements publics.

Source : COI 2006, statistique publique, DGAFP-Dares-CEE. 
Ce « privilège de l'âge » propre aux agents de l'État semble cependant provenir d'un effet de composition : lorsqu'on prend en compte la catégorie socioprofessionnelle, le niveau d'études et les autres variables incluses dans les modèles, la probabilité d'une utilisation variée ou intermédiaire est plus élevée entre trente et cinquante ans dans les secteurs marchands, alors qu'elle varie peu avec l'âge dans la fonction publique de 1'État (cf. tableaux 5 et 6).

De même, une ancienneté d'au moins quinze années augmente plus significativement la probabilité d'une utilisation intermédiaire dans les

Tableau 5

Modélisation des seuils d'utilisation de l'informatique : utilisation intermédiaire et utilisation variée Secteurs marchands

\begin{tabular}{|c|c|c|c|}
\hline & & \multicolumn{2}{|c|}{$\begin{array}{l}\text { Utilisation intermédiaire et variée versus } \\
\text { pas d'utilisation et utilisation basique }\end{array}$} \\
\hline & & MODELE 2Ma & MODELE $2 \mathrm{Mb}$ \\
\hline \multicolumn{2}{|l|}{ Constante } & $-1,04^{\star \star \star}(0,105)$ & $-1,20^{\star \star \star}(0,121)$ \\
\hline \multirow[t]{2}{*}{ Secteur d'activité de l'entreprise } & Commerce & $-0,92^{* \star *}(0,073)$ & $-0,92^{* \star *}(0,073)$ \\
\hline & Construction-transport & $-0,21^{\star \star}(0,087)$ & $-0,16 *(0,087)$ \\
\hline \multirow[t]{2}{*}{ Réf. : industrie } & Finance & 0,34 *** $(0,096)$ & $0,35^{\star \star \star}(0,097)$ \\
\hline & Services aux entreprises & 0,02 n.s. $(0,082)$ & 0,08 n.s. $(0,083)$ \\
\hline \multicolumn{4}{|l|}{ L'entreprise appartient à un groupe } \\
\hline Réf. : non & Groupe & $0,37^{\star \star \star}(0,061)$ & $0,33^{\star \star \star}(0,062)$ \\
\hline \multicolumn{4}{|l|}{ L'entreprise est équipée en ERP } \\
\hline Réf. : non & ERP & 0,22 *** $(0,055)$ & $0,21^{\star \star \star}(0,056)$ \\
\hline \multicolumn{4}{|l|}{$\begin{array}{l}\text { L'entreprise est équipée en outils } \\
\text { de travail collaboratif }\end{array}$} \\
\hline Réf. : non & Travail collaboratif & $0,50 * * *(0,056)$ & $0,45^{\star \star \star}(0,057)$ \\
\hline \multirow[t]{2}{*}{ Fonction du salarié } & Production, nettoyage, manutention & $-1,19^{\star \star \star}(0,078)$ & $-1,20$ *** $(0,079)$ \\
\hline & Gestion, comptabilité & $0,59^{\star \star \star}(0,093)$ & 0,60 *** $(0,093)$ \\
\hline \multirow[t]{2}{*}{ Réf. : Secrétariat, saisie, commercial } & Études, R\&D, enseignement, soin & - 0,17 n.s. $(0,117)$ & - 0,16 n.s. $(0,118)$ \\
\hline & Autres fonctions. & $-0,53^{\star \star \star}(0,094)$ & $-0,53^{\star \star \star}(0,094)$ \\
\hline PCS & Cadres & $2,45^{\star \star \star}(0,109)$ & $2,45^{\star \star \star}(0,109)$ \\
\hline \multirow[t]{2}{*}{ Réf. : employé } & Professions. intermédiaires & 0,91 *** $(0,069)$ & 0,91 *** $(0,069)$ \\
\hline & Ouvriers & $-1,61^{\star \star \star}(0,093)$ & $-1,57^{\star * \star}(0,094)$ \\
\hline \multicolumn{4}{|l|}{ Sexe } \\
\hline Réf. : femme & Homme & $0,14^{\star \star}(0,061)$ & $0,13^{\star \star}(0,061)$ \\
\hline Diplôme & Supérieur ou égal au bac & $1,52^{\star \star \star}(0,067)$ & $1,53^{\star \star \star}(0,068)$ \\
\hline Réf. : primaire ou technique court & Secondaire & $0,77^{\star \star \star}(0,066)$ & $0,78^{\star \star \star}(0,067)$ \\
\hline Âge & Moins de 30 ans & $-0,17^{*}(0,088)$ & \\
\hline \multirow[t]{2}{*}{ Réf. : 50 ans et plus } & $30-39$ ans & 0,14 * $(0,076)$ & \\
\hline & $40-49$ ans & $0,15 *(0,073)$ & \\
\hline \multirow[t]{3}{*}{ Âge et ancienneté } & Moins de 40 ans, ancienneté $<15$ ans & & $-0,16^{*}(0,090)$ \\
\hline & Moins de 40 ans, ancienneté $>=15$ ans & & $0,22^{*}(0,126)$ \\
\hline & $40-49$ ans, ancienneté $<15$ ans & & - 0,16 n.s. $(0,106)$ \\
\hline \multirow{2}{*}{$\begin{array}{l}\text { Réf. : } 50 \text { ans et plus, ancienneté } \\
\text { supérieure à } 15 \text { ans }\end{array}$} & $40-49$ ans, ancienneté $>=15$ ans & & 0,21 ** $(0,095)$ \\
\hline & $>=50$ ans, ancienneté $<15$ ans & & - 0,15 n.s. $(0,118)$ \\
\hline Score d'entraide & Moyenne & & $0,32^{\star \star \star}(0,064)$ \\
\hline Réf. : entraide basse & Élevée & & $0,55^{\star * \star}(0,069)$ \\
\hline
\end{tabular}

Lecture : le modèle 2Ma présente la probabilité d'une utilisation intermédiaire ou variée de l'informatique versus l'absence d'utilisation ou une utilisation basique dans les secteurs marchands (voir encadré 2) avant introduction de l'ancienneté et de l'entraide ; $2 \mathrm{Mb}$ après l'introduction de ces variables. La situation de référence est signalée dans la première colonne. Les coefficients sont issus de l'estimation des modèles Logit. Les écarts-types sont présentés entre parenthèses.

Dans les secteurs marchands, la probabilité d'avoir une utilisation intermédiaire ou variée de l'informatique (plutôt que pas d'utilisation ou un utilisation basique) est significativement moins élevée pour un salarié âgé de moins de 30 ans que pour un salarié de 50 ans et plus. n.s. non significatif ; ${ }^{* *}$ significatif au seuil de $1 \%$, ${ }^{* *}$ significatif au seuil de $5 \%,{ }^{*}$ significatif au seuil de $10 \%$.

Champ : salariés des entreprises marchandes non agricoles de plus de 20 salariés.

Sources : fichier couplé dans la fonction publique de l'État, enquête COI couplée 2006, statistique publique, DGAFP - CEE. 
entreprises que dans la fonction publique de l'État. Dans tous les secteurs, l'utilisation variée de l'informatique ne semble en revanche dépendre ni de l'âge, ni de l'ancienneté (cf. tableaux 7 et 8 ) : elle est surtout dépendante du niveau de diplôme et de la catégorie socioprofessionnelle. La proportion des salariés dont le niveau d'utilisation de l'informatique est intermédiaire, et plus encore variée, est ainsi nettement supérieure parmi les cadres que parmi les ouvriers ou employés.

Dans tous les secteurs, les fonctions commerciales, de secrétariat, de comptabilité et de gestion ou les fonctions d'étude et de recherche génèrent une utilisation moins basique de l'informatique. La présence d'outils de travail collaboratifs accroît la variété d'utilisation des outils informatiques par les salariés - c'est aussi le cas des ERP dans les entreprises. Mais dans la fonction publique de l'État, la présence de ces progiciels augmente peu significativement la probabilité d'utilisation intermédiaire (par rapport à pas d'utilisation ou une utilisation basique), et diminue celle d'une utilisation variée. L'utilisation des ERP y est en effet relativement banale, les agents alimentant ou consultant

Tableau 6

Modélisation des seuils d'utilisation de l'informatique : utilisation intermédiaire et utilisation variée Fonction publique de l'État

\begin{tabular}{|c|c|c|c|}
\hline & & \multicolumn{2}{|c|}{$\begin{array}{l}\text { Utilisation intermédiaire et variée versus } \\
\text { pas d'utilisation et utilisation basique }\end{array}$} \\
\hline & & MODELE 2Pa & MODELE 2Pb \\
\hline Constante & & $0,68^{\star \star}(0,285)$ & 0,40 n.s. $(0,349)$ \\
\hline $\begin{array}{l}\text { L'administration est équipée en ERP } \\
\text { Réf. : non }\end{array}$ & ERP & $0,35 *(0,185)$ & $0,35^{*}(0,188)$ \\
\hline $\begin{array}{l}\text { L'administration est équipée en } \\
\text { outils de travail collaboratif } \\
\text { Réf. : non }\end{array}$ & Travail collaboratif & 0,04 n.s. $(0,196)$ & 0,06 n.s. $(0,198)$ \\
\hline $\begin{array}{l}\text { Fonction du salarié } \\
\text { Réf. : secrétariat, saisie, commercial }\end{array}$ & $\begin{array}{l}\text { Production, nettoyage, manutention } \\
\text { Gestion comptabilité } \\
\text { Études, R\&D, enseignement, soin } \\
\text { Autre fonction }\end{array}$ & $\begin{array}{l}-2,87^{\star * \star}(0,326) \\
-0,10 \text { n.s. }(0,334) \\
-0,97^{\star *}(0,384) \\
-1,80^{* \star *}(0,299)\end{array}$ & $\begin{array}{l}-2,73^{\star \star \star}(0,337) \\
-0,15^{\text {n.s. }}(0,339) \\
-0,91^{\star \star}(0,395) \\
-1,95^{\star \star \star}(0,305)\end{array}$ \\
\hline $\begin{array}{l}\text { PCS } \\
\text { Réf. : employé }\end{array}$ & $\begin{array}{l}\text { Cadres } \\
\text { Professions intermédiaires } \\
\text { Ouvriers }\end{array}$ & $\begin{array}{l}2,27^{\star \star \star}(0,388) \\
1,64^{\star \star \star}(0.259) \\
0,27 \text { n.s. }(0,342)\end{array}$ & $\begin{array}{l}2,26^{\star \star \star}(0,393) \\
1,69^{\star \star \star}(0,268) \\
0,18 \text { n.s. }(0,351)\end{array}$ \\
\hline $\begin{array}{l}\text { Sexe } \\
\text { Réf. : femme }\end{array}$ & Homme & 0,28 n.s. $(0,210)$ & 0,21 n.s. $(0,215)$ \\
\hline $\begin{array}{l}\text { Diplôme } \\
\text { Réf. : primaire ou technique court }\end{array}$ & $\begin{array}{l}\text { Secondaire général } \\
\text { Supérieur ou égal au bac }\end{array}$ & $\begin{array}{l}0,97^{* * *}(0,238) \\
0,83^{\star *}(0,263)\end{array}$ & $\begin{array}{l}0,92^{* \star *}(0,245) \\
0,81^{\star \star *}(0,272)\end{array}$ \\
\hline $\begin{array}{l}\text { Âge } \\
\text { Réf. : } 50 \text { ans et plus }\end{array}$ & $\begin{array}{l}\text { Moins de } 30 \text { ans. } \\
30-39 \text { ans } \\
40-49 \text { ans }\end{array}$ & $\begin{array}{l}-0,52 \text { n.s. }(0,469) \\
-0,22 \text { n.s. }(0,264) \\
0,30 \text { n.s. }(0,220)\end{array}$ & \\
\hline $\begin{array}{l}\text { Âge et ancienneté } \\
\text { Réf. : } 50 \text { ans et plus, ancienneté } \\
\text { supérieure à } 15 \text { ans }\end{array}$ & $\begin{array}{l}\text { Moins de } 40 \text { ans, ancienneté }<15 \text { ans } \\
\text { Moins de } 40 \text { ans, ancienneté >=15 ans. } \\
40-49 \text { ans, ancienneté }<15 \text { ans } \\
40-49 \text { ans, ancienneté }>=15 \text { ans } \\
>=50 \text { ans, ancienneté }<15 \text { ans }\end{array}$ & & $\begin{array}{l}-0,43 \text { n.s. }(0,296) \\
-0,92 *(0,538) \\
-0,36 \text { n.s. }(0,352) \\
0,33 \text { n.s. }(0,270) \\
-0,52 \text { n.s. }(0,324)\end{array}$ \\
\hline $\begin{array}{l}\text { Score d'entraide } \\
\text { Réf. : entraide basse }\end{array}$ & $\begin{array}{l}\text { Moyenne } \\
\text { Élevée }\end{array}$ & & $\begin{array}{l}0,63^{\star \star \star}(0,233) \\
0,79^{\star \star \star}(0,255)\end{array}$ \\
\hline
\end{tabular}

Lecture : le modèle 2Pa présente la probabilité d'une utilisation intermédiaire ou variée de l'informatique versus l'absence d'utilisation ou une utilisation basique dans la fonction publique de l'État (voir encadré 2) avant introduction de l'ancienneté et de l'entraide ; $2 P b$ après l'introduction de ces variables. La situation de référence est signalée dans la première colonne. Les coefficients sont issus de l'estimation des modèles Logit. Les écarts-types sont présentés entre parenthèses.

Dans la fonction publique de l'Etat la probabilité d'avoir une utilisation intermédiaire ou variée de l'informatique (plutôt que pas d'utilisation ou une utilisation basique) n'est pas significativement différente pour un agent de moins de 30 ans que pour un agent de 50 ans et plus.

n.s. non significatif ; *** significatif au seuil de $1 \%$, ** significatif au seuil de $5 \%$, ${ }^{*}$ significatif au seuil de $10 \%$.

Champ : fonction publique de l'État hors enseignants, magistrats, agents du ministère de la Défense et des établissements publics.

Sources : fichier couplé dans la fonction publique de l'État, enquête COI couplée 2006, statistique publique, DGAFP - CEE. 
les bases de données à l'aide de ces nouveaux équipements de manière plus standardisée, là où ils le faisaient auparavant à partir d'applications dédiées éventuellement développées localement. Dans les entreprises marchandes en revanche, c'est souvent par le biais d'un ERP (utilisé par exemple pour la gestion des stocks) que l'informatique pénètre dans les ateliers, les entrepôts et les magasins.

Au total, les salariés les plus âgés des entreprises marchandes apparaissent encore relativement sous-équipés alors que les outils informatiques sont désormais largement répandus. $\mathrm{Si}$

Tableau 7

Modélisation des seuils d'utilisation de l'informatique : utilisation variée ou non

Secteurs marchands

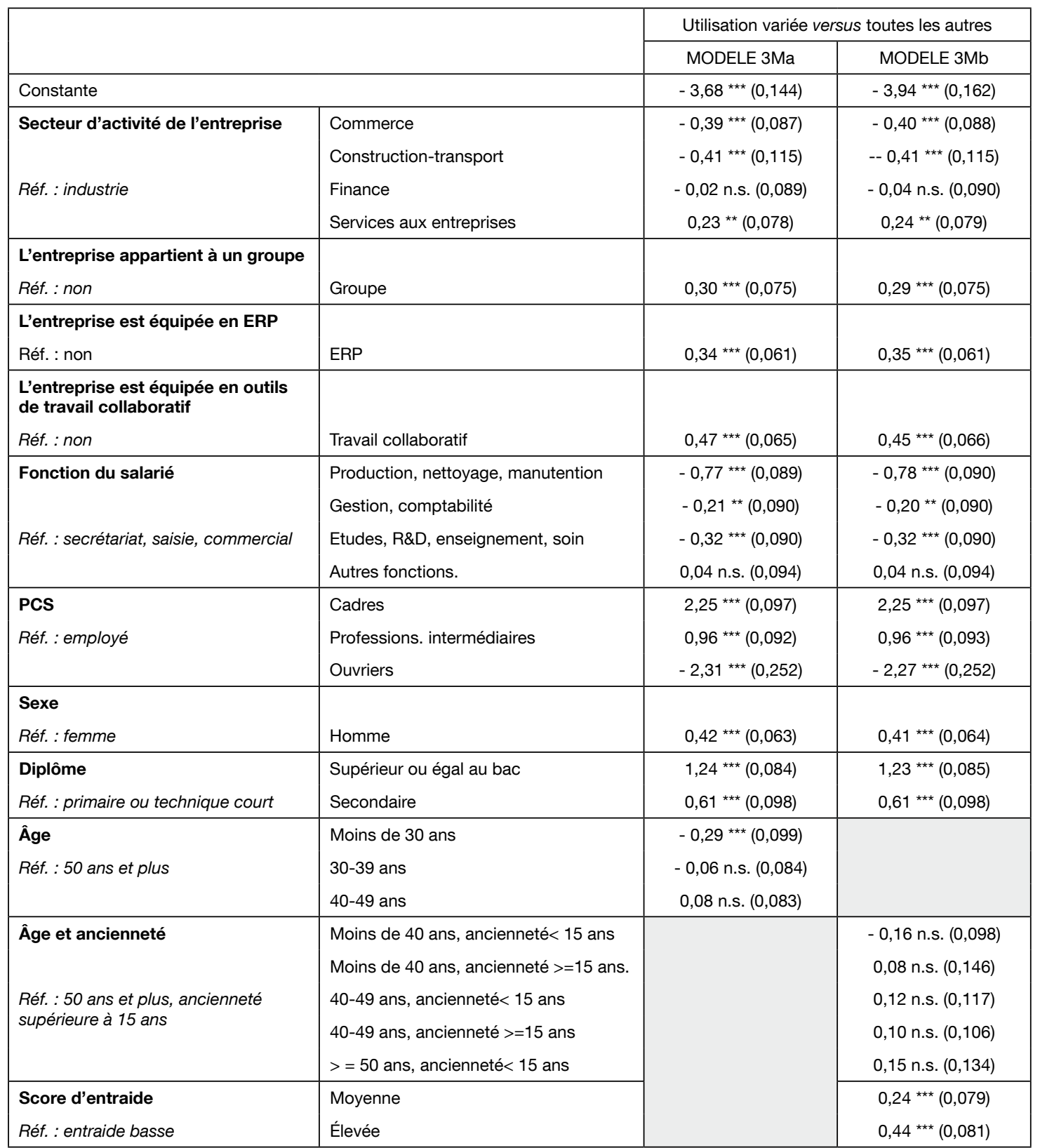

Lecture : le modèle 3Ma présente la probabilité d'une utilisation variée de l'informatique versus toutes les autres dans les secteurs marchands (voir encadré 2) avant introduction de l'ancienneté et de l'entraide ; 3Mb après l'introduction de ces variables. La situation de référence est signalée dans la première colonne. Les coefficients sont issus de l'estimation des modèles Logit. Les écarts-types sont présentés entre parenthèses.

Dans les secteurs marchands, la probabilité d'une utilisation variée de l'informatique (plutôt que d'autres niveaux d'utilisation) est significativement moins élevée pour un salarié âgé de moins de 30 ans que pour un salarié de 50 ans et plus.

n.s. non significatif ; ${ }^{* * *}$ significatif au seuil de $1 \%$, ${ }^{* *}$ significatif au seuil de $5 \%$, ${ }^{*}$ significatif au seuil de $10 \%$.

Champ : salariés des entreprises marchandes non agricoles de plus de 20 salariés.

Sources : fichier couplé dans la fonction publique de l'État, enquête COI couplée 2006, statistique publique, DGAFP - CEE. 
l'on adhère à l'interprétation en termes d'effet de génération, formulée à la fin des années 1980 lorsque l'informatisation du travail commençait à prendre de l'ampleur, il apparaît que des salariés ayant commencé à travailler dans un univers non informatisé et qui n'ont pas été licenciés, mis en préretraite ou en retraite, peuvent encore occuper des postes sans informatique, là où leurs collègues plus jeunes utilisent ces outils (10). L'âge ne semble cependant pas avoir d'impact sur l'utilisation diversifiée d'outils tels que l'Internet ou l'intranet, le remplissage en ligne de formulaires administratifs ou la connexion à distance au système d'information de l'entreprise. Ce résultat portant sur l'usage professionnel de l'informatique dans le secteur privé rejoint certaines analyses sur l'usage domestique d'Internet : "l'effet de génération, $s$ 'il explique le contact avec la technique, ne

10. Les générations qui utilisaient très peu l'informatique au début des années quatre-vingt sont généralement parties à la retraite. Selon Rouquette (1999), les salariés nés après 1968 ont le plus souvent appris l'informatique au cours de leur enfance ou de leur scolarité. La majorité de leurs aînés a découvert l'informatique au cours de la vie professionnelle, mais ceux qui avaient plus de 50 ans en 1998 (date de l'enquête exploitée par Céline Rouquette) n'avaient guère eu l'occasion d'apprendre l'informatique ni à l'école, ni au travail. En 2006, ils auraient eu plus de 58 ans.

Tableau 8

Modélisation des seuils d'utilisation de l'informatique : utilisation variée

Fonction publique de l'État

\begin{tabular}{|c|c|c|c|}
\hline & & \multicolumn{2}{|c|}{ Utilisation variée versus toutes les autres } \\
\hline & & MODELE 3Pa & MODELE 3Pb \\
\hline Constante & & $-2,97^{\star \star \star}(0,383)$ & $-3,27^{* \star \star}(0,450)$ \\
\hline $\begin{array}{l}\text { L'administration est équipée en ERP } \\
\text { Réf. : non }\end{array}$ & ERP & $-0,47^{\star *}(0,200)$ & $-0,49^{* *}(0,201)$ \\
\hline $\begin{array}{l}\text { L'administration est équipée en } \\
\text { outils de travail collaboratif } \\
\text { Réf. : non }\end{array}$ & Travail collaboratif & 0,49 ** $(0,230)$ & $0,55^{\star *}(0,234)$ \\
\hline $\begin{array}{l}\text { Fonction du salarié } \\
\text { Réf. : secrétariat, saisie, commercial }\end{array}$ & $\begin{array}{l}\text { Production, nettoyage, manutention } \\
\text { Gestion comptabilité } \\
\text { Études, R\&D, enseignement, soin } \\
\text { Autre fonction }\end{array}$ & $\begin{array}{l}-0,80^{*}(0,414) \\
-0,82^{* *}(0,338) \\
0,26 \text { ns }(0,349) \\
-0,77^{\star *}(0,342)\end{array}$ & $\begin{array}{l}-0,73^{*}(0,417) \\
-0,81^{\star *}(0,340) \\
0,27 \text { n.s. }(0,352) \\
-0,72^{* *}(0,345)\end{array}$ \\
\hline $\begin{array}{l}\text { PCS } \\
\text { Réf. : employé }\end{array}$ & $\begin{array}{l}\text { Cadres } \\
\text { Professions intermédiaires } \\
\text { Ouvriers }\end{array}$ & $\begin{array}{c}1,96^{* \star *}(0,317) \\
0,77^{\star \star}(0,285) \\
0,43 \text { n.s. }(0,553)\end{array}$ & $\begin{array}{c}1,97^{\star * \star}(0,318) \\
0,80^{* \star}(0,287) \\
0,35 \text { n.s. }(0,558)\end{array}$ \\
\hline $\begin{array}{l}\text { Sexe } \\
\text { Réf. : femme }\end{array}$ & Homme & $0,37^{*}(0,213)$ & 0,32 n.s. $(0,215)$ \\
\hline $\begin{array}{l}\text { Diplôme } \\
\text { Réf. : primaire ou technique court }\end{array}$ & $\begin{array}{l}\text { Supérieur ou égal au bac } \\
\text { Secondaire général }\end{array}$ & $\begin{array}{l}1,15^{* \star *}(0,329) \\
0,81^{\star *}(0,337)\end{array}$ & $\begin{array}{l}1,08^{* \star *}(0,333) \\
0,75^{\star *}(0,339)\end{array}$ \\
\hline $\begin{array}{l}\text { Âge } \\
\text { Réf. : } 50 \text { ans et plus }\end{array}$ & $\begin{array}{l}\text { Moins de } 30 \text { ans } \\
30-39 \text { ans } \\
40-49 \text { ans }\end{array}$ & $\begin{array}{l}0,09 \text { n.s. }(0,506) \\
-0,41 \text { n.s. }(0,285) \\
\text { - } 0,07 \text { n.s. }(0,225)\end{array}$ & \\
\hline $\begin{array}{l}\text { Âge et ancienneté } \\
\text { Réf. : } 50 \text { ans et plus, ancienneté supé- } \\
\text { rieure à } 15 \text { ans }\end{array}$ & $\begin{array}{l}\text { Moins de } 40 \text { ans, ancienneté }<15 \text { ans } \\
\text { Moins de } 40 \text { ans, ancienneté }>=15 \text { ans } \\
40-49 \text { ans, ancienneté }<15 \text { ans } \\
40-49 \text { ans, ancienneté }>=15 \text { ans } \\
>=50 \text { ans, ancienneté }<15 \text { ans }\end{array}$ & & $\begin{array}{r}-0,26 \text { n.s. }(0,290) \\
-1,49 \text { n.s. }(1,127) \\
0,26 \text { n.s. }(0,340) \\
-0,18 \text { n.s. }(0,276) \\
0,06 \text { n.s. }(0,331)\end{array}$ \\
\hline $\begin{array}{l}\text { Score d'entraide } \\
\text { Réf. : entraide basse }\end{array}$ & $\begin{array}{l}\text { Moyenne } \\
\text { Élevée }\end{array}$ & & $\begin{array}{c}0,29 \text { n.s. }(0,266) \\
0,46 *(0,276)\end{array}$ \\
\hline
\end{tabular}

Lecture : le modèle 3Pa présente la probabilité d'une utilisation variée de l'informatique versus toutes les autres dans la fonction publique de l'État (voir encadré 2) avant introduction de l'ancienneté et de l'entraide; 3Pb après l'introduction de ces variables. La situation de référence est signalée dans la première colonne. Les coefficients sont issus de l'estimation des modèles Logit. Les écarts-types sont présentés entre parenthèses.

Dans la fonction publique de l'Etat la probabilité d'avoir une utilisation variée de l'informatique (plutôt que d'autres utilisations) n'est pas significativement différente pour un agent de moins de 30 ans que pour un agent de 50 ans et plus.

n.s. non significatif ; ${ }^{* *}$ significatif au seuil de $1 \%,{ }^{* *}$ significatif au seuil de $5 \%,{ }^{*}$ significatif au seuil de $10 \%$

Champ : fonction publique de l'État hors enseignants, magistrats, agents du ministère de la Défense et des établissements publics.

Sources : fichier couplé dans la fonction publique de l'État, enquête COI couplée 2006, statistique publique, DGAFP - CEE. 
rend pas ou peu compte de l'intensité des usages » (Moatty, 2009).

Dans la fonction publique de l'État en revanche, les quinquagénaires n'ont pas moins accès que les plus jeunes aux outils informatiques, et en font tout autant un usage varié. Le mode de recrutement dans la fonction publique de l'État, précoce et par concours, permettrait-il de sélectionner des agents plus adaptables que les salariés recrutés dans le secteur marchand? L'hypothèse est difficilement défendable, et elle ne peut pas être testée sur le plan empirique. Un registre hypothétique alternatif peut être bâti à la lecture de travaux ergonomiques et sociologiques.

\section{La formation professionnelle et l'entraide comme facteurs d'évolution des seniors}

D es études de cas fondées sur des observations enrichies par des entretiens ont en effet montré que la capacité d'adaptation des seniors variait considérablement, principalement en fonction de la manière dont l'organisation tire profit de l'expérience de ses employés, dispense des formations, mais aussi dont le collectif mobilise l'entraide (Gaudart, 2000). Selon Cau-Bareille et Volkoff (1998), il n'y a pas de fatalité aux difficultés d'accès des plus âgés à l'informatisation. Les formations régulières et de longue durée permettent aux salariés qui ne se sont pas familiarisés avec l'ordinateur dès leur scolarité ou dans les débuts de leur vie active d'atteindre de hauts niveaux de performance. De telles formations paraissent même particulièrement profitables aux seniors (Delgoulet et Gonon, 2000 ; Cau-Bareille et Volkoff, 1998). Comme l'a montré une analyse fondée sur l'enquête COI 1996 : «les efforts de formation continue réalisés par les entreprises apparaissent donc potentiellement susceptibles de contrebalancer les effets de biais à l'encontre de l'âge associés à la diffusion des nouvelles technologies et des pratiques de travail innovantes » (Behagel et al., 2009). Devant s'adapter à la main-d'œuvre présente, majoritairement vieillissante, l'organisation de l'État met en effet en œuvre ces formes de régulation spécifiques.

La probabilité d'être formé à l'informatique est plus élevée dans la fonction publique de l'État, notamment pour les seniors

Le questionnaire COI 2006 détaille la spécialité, la date et la durée des formations reçues par les salariés dans leur entreprise ou administration. Les agents de la fonction publique de l'État sont, en général, plus souvent et plus longtemps formés que les salariés des secteurs marchands (Perez, 2002). Selon COI, $58 \%$ des agents de la fonction publique de l'État ont suivi une formation en 2005-2006 contre $53 \%$ des salariés de l'ensemble des secteurs marchands, l'écart se creusant pour les seules formations en informatique (respectivement $27 \%$ et $19 \%$ ). En tenant compte des formations reçues dans le passé, $60 \%$ des agents de la fonction publique de l'État ont bénéficié, au moins une fois au cours de leur carrière, d'une formation à l'informatique proposée par leur employeur actuel. Cette proportion n'est que de $38 \%$ dans l'ensemble des secteurs marchands.

Dans tous les secteurs, la proportion de salariés formés chaque année, toutes spécialités confondues, diminue sensiblement après 50 ans (Lainé, 2003). La proportion de seniors formés récemment est néanmoins plus élevée dans la fonction publique de l'État (49\%) que dans l'ensemble des secteurs marchands (42\%) (cf. graphique V). Si l'on s'en tient aux formations à l'informatique, les seniors de la fonction publique de l'État ont été autant formés en 20052006 que les agents trentenaires, alors que dans les secteurs marchands l'accès à ces formations décroît légèrement avec l'âge (cf. graphique VI). En tenant compte de l'historique des formations informatiques reçues par les salariés dans leur emploi actuel, la proportion des salariés formés augmente avec l'âge dans tous les secteurs, mais le phénomène est à nouveau particulièrement marqué dans la fonction publique de l'État : $64 \%$ des seniors de l'État ont reçu au moins une fois, dans un passé plus ou moins récent, une formation à l'informatique, contre $44 \%$ dans l'ensemble des secteurs marchands.

Les agents de la fonction publique de l'État, particulièrement les seniors, ont enfin non seulement été plus souvent formés à l'informatique que les autres salariés, mais ils l'ont aussi été plus longuement : $17 \%$ des formations informatiques suivies par les agents de l'État ont duré au moins un mois, contre $7 \%$ des formations suivies par les salariés de l'ensemble des secteurs marchands. Après 50 ans, ces taux atteignent respectivement $21 \%$ et $11 \%$.

Ainsi, particulièrement nombreux et non « sélectionnés » selon leur aptitude supposée à s'adapter aux nouvelles technologies, les seniors de la fonction publique de l'État ont généralement été formés à l'informatique, au moment de l'intro- 
duction des ordinateurs dans l'administration ou plus tard, à l'occasion de l'arrivée d'un nouveau logiciel, favorisant leur accès à des postes de travail informatisés. La formation dispensée tout au long de la carrière, paraît ainsi jouer le rôle attendu. Ce surcroît de formation des seniors de la fonction publique aurait pu être un effet de la promotion à l'ancienneté, et découler de leur profil plus qualifié (relativement aux salariés des autres secteurs). Mais leur égale probabilité d'accès à la formation perdure en contrôlant la catégorie socioprofessionnelle.

Toutes choses égales par ailleurs, les agents de la fonction publique de l'État ont davantage été formés à l'informatique par leur employeur, en 2005 et 2006 et tout au long de leur carrière (cf. tableau 9, modèles 4MP et 5MP). Dans ce secteur, il n'y a pas de différence très significative selon l'âge. Les agents de la fonction publique de l'État suivent aussi plus souvent des formations d'une durée supérieure ou égale à un mois (cf. tableau 10, modèles 4P et 5P). Enfin, quel que soit le secteur, les agents plus âgés, et ceux ayant une plus grande ancienneté suivent davantage des formations de longue durée. Le rôle de l'ancienneté dans l'accès à la formation semble donc aller au-delà de l'effet du cumul (plus on est ancien, plus on a eu d'occasion de se former), puisqu'elle permet d'accéder à des formations plus longues. On peut faire ici l'hypothèse que les salariés mobiles sur le marché du travail qui, à âge donné, sont moins anciens dans l'entreprise ou l'administration, ont été recrutés en tenant compte des connaissances déjà acquises dans des précédents emplois, si bien que les formations qu'ils reçoivent seraient davantage tournées vers une simple adaptation au poste. Ainsi, la formation en interne jouerait bien son rôle de mise à niveau des compétences nécessaires à la maîtrise de l'outil, tout au moins du point de vue de l'âge.

Les salariés les plus qualifiés ont par ailleurs une plus grande probabilité d'être formés à l'informatique, particulièrement les cadres - résultat régulièrement vérifié au sujet de la formation en général (Blasco et al., 2009). Le poids de la hiérarchie scolaire est en revanche moins net, car si un niveau de formation initiale primaire ou technique court diminue la probabilité d'accès à une formation en informatique (et aussi, on

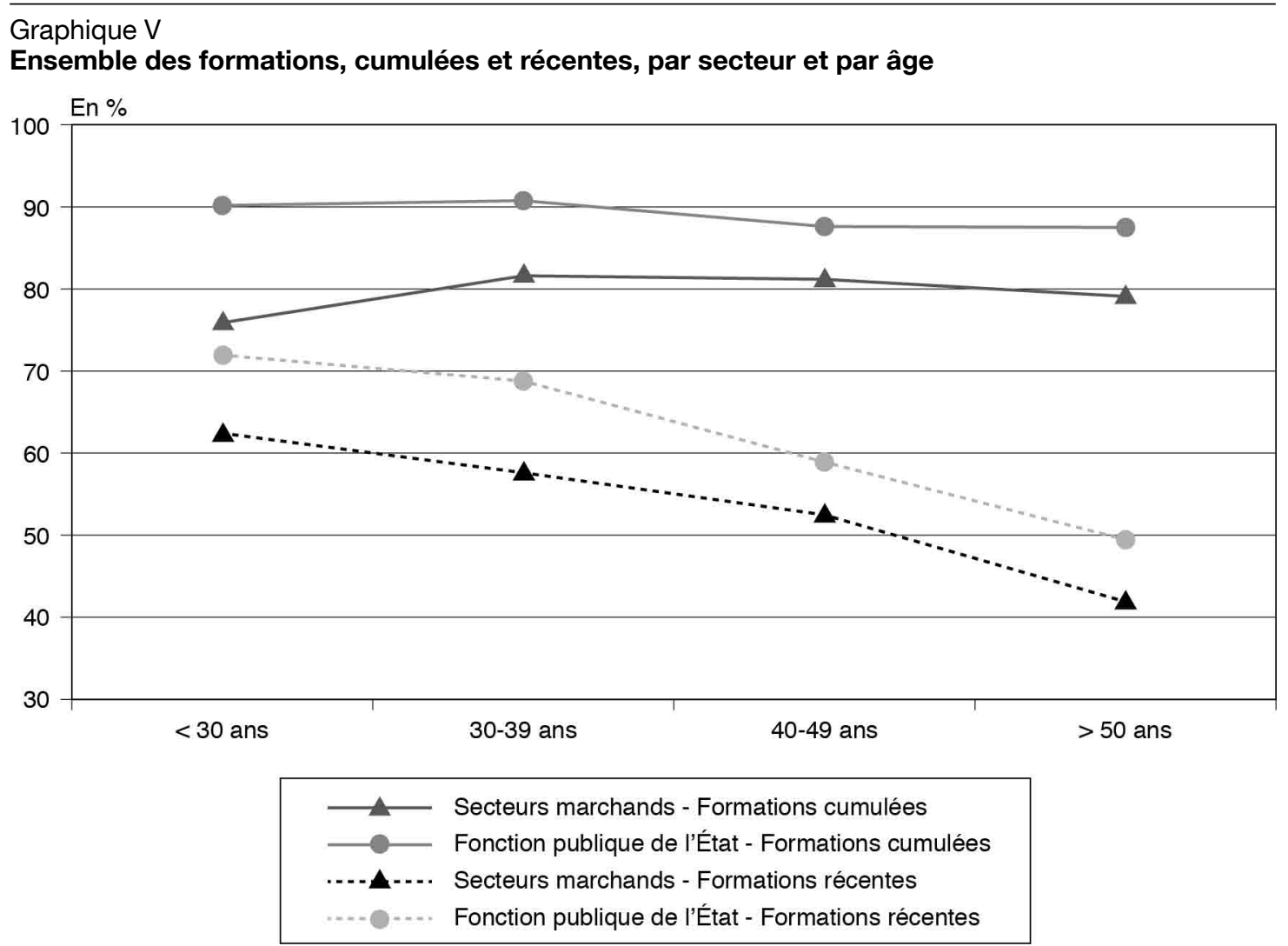

Lecture : $90 \%$ des agents de la fonction publique de l'État âgés de moins de 30 ans déclarent avoir été formés au moins une fois à l'informatique par leur employeur, et 71,9\% déclarent avoir été formés récemment (en 2005 ou 2006).

Champ : fonction publique de l'État hors enseignants, magistrats, agents du ministère de la Défense et des établissements publics. Pour les secteurs marchands : salariés des entreprises marchandes non agricoles de plus de 20 salariés.

Sources : COI 2006, statistique publique, DGAFP-Dares-CEE, données pondérées. 
l'a vu, celle d'accès à l'outil), avoir un niveau supérieur au bac n'augmente pas les chances d'être formé par rapport à un niveau secondaire. Exercer des fonctions parmi les plus informatisées (comptabilité et gestion, fonctions commerciales et secrétariat), augmente évidemment la probabilité d'être formé à l'informatique.

\section{L'entraide au sein du collectif pourrait favoriser l'accès aux outils informatiques}

Entraide et niveau d'utilisation de l'informatique augmentent ensemble quel que soit l'âge, mais plus encore pour les salariés âgés (cf. graphique VII). Ce lien confirme l'importance de la « solidarité technique » pour la maîtrise des outils en général et de l'informatique en parti- culier (Dodier, 1995 et 1997 ; Gollac, 1996), d'autant plus que les nouveaux outils collaboratifs ouvrent des opportunités d'échange et donc d'entraide.

Le niveau d'entraide, mesuré par un indicateur composite du soutien apporté par le collectif et du besoin individuel d'y recourir (cf. annexe 2) baisse néanmoins avec l'âge dans tous les secteurs. Les débutants déclarent davantage recevoir de l'aide du collectif que les anciens : dans la suite de la carrière, la maîtrise des tâches et des missions permet vraisemblablement d'acquérir plus d'autonomie, rendant le support du collectif moins nécessaire - sauf pour s'approprier de nouvelles techniques et notamment pour une utilisation plus avancée de l'informatique.

Graphique VI

Formations en informatique : cumulées, récentes et longues, par secteur et par âge

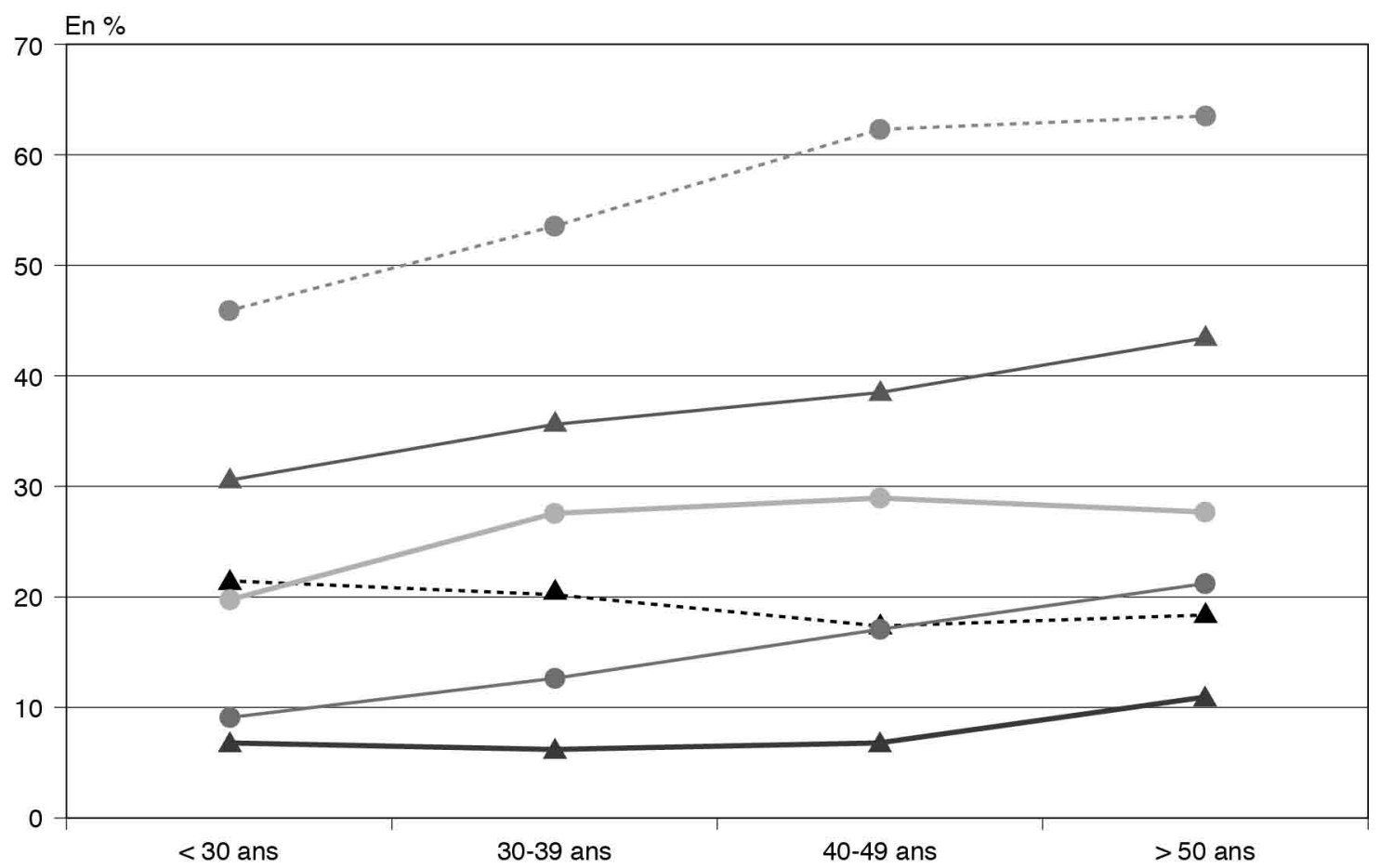

Secteurs marchands - Formations cumulées à l'informatique

Lecture : 46,1 \% des agents de la fonction publique de l'État âgés de moins de 30 ans déclarent avoir été formés au moins une fois à l'informatique par leur employeur; 19,9\% déclarent avoir été formés à l'informatique récemment (en 2005 ou 2006); et 9,8 \% avoir suivi une formation longue (d'au moins 30 jours).

Champ : fonction publique de l'État hors enseignants, magistrats, agents du ministère de la Défense et des établissements publics. Pour les secteurs marchands : salariés des entreprises marchandes non agricoles de plus de 20 salariés.

Sources : COI 2006, statistique publique, DGAFP-Dares-CEE, données pondérées. 
L'introduction de l'indicateur de niveau d'entraide dans les modèles d'usage de l'informatique (cf. tableaux 3 à 8) confirme la corrélation positive, dans le public et le privé, entre l'utilisation de l'informatique (quel qu'en soit le niveau) et l'entraide, lorsque les autres variables sont contrôlées. De façon complémentaire à la formation professionnelle, l'entraide pourrait ainsi représenter un facteur déterminant pour « égaliser 》 les chances des salariés dans leur accès à l'informatique.

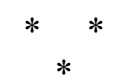

L'utilisation professionnelle de l'informatique est devenue incontournable, y compris pour la génération des quinquagénaires qui l'a découverte au travail dans les années 1980-1990. Si les générations de salariés n'ayant eu aucun contact avec l'informatique durant la majeure partie de leur carrière sont maintenant pour l'essentiel parties à la retraite, une forme de " discrimination technologique » à l'encontre des seniors semble néanmoins subsister dans les secteurs marchands, en sus des effets de sélection de la main-d'œuvre dans les entreprises les plus informatisées mis en évidence dans diffé-

Tableau 9

Formations à l'informatique selon des critères personnels et professionnels Ensemble des secteurs (État et entreprises appartenant à un groupe)

\begin{tabular}{|c|c|c|c|c|}
\hline & Modèle 4MP & Modèle 5MP & Modèle 6MP \\
\hline & & $\begin{array}{l}\text { Cumul formation } \\
\text { informatique chez } \\
\text { l'employeur actuel }\end{array}$ & $\begin{array}{l}\text { Formation } \\
\text { informatique en } \\
2005-2006\end{array}$ & $\begin{array}{l}\text { Formation à } \\
\text { l'informatique } \\
\text { d'au moins 30h }\end{array}$ \\
\hline \multicolumn{2}{|l|}{ Constante } & $0,25^{\star *}(0,098)$ & $-1,61^{\star \star \star}(0,113)$ & $-2,32^{* \star *}(0,161)$ \\
\hline \multirow[t]{2}{*}{ Secteur } & Construction et transports & $-0,50^{* \star \star}(0,081)$ & $-0,22$ ** $(0,099)$ & $-0,30$ ** $(0,155)$ \\
\hline & Commerce & $-0,40^{\star \star \star}(0,067)$ & - 0,02 n.s. $(0,076)$ & $-0,57^{\star \star \star}(0,131)$ \\
\hline \multirow[t]{3}{*}{ Réf. : industrie } & Activités financières & - 0,10 n.s. $(0,080)$ & 0,11 n.s. $(0,084)$ & 0,10 n.s. $(0,118)$ \\
\hline & Services aux entreprises & $-0,42^{\star \star \star}(0,070)$ & $-0,10$ n.s. $(0,078)$ & 0,00 n.s. $(0,113)$ \\
\hline & Fonction publique de l'État & $0,19 * *(0,087)$ & $0,27^{\star \star *}(0,091)$ & $0,47^{\star \star \star}(0,117)$ \\
\hline \multirow{2}{*}{$\begin{array}{l}\text { Outils présents dans l'entre- } \\
\text { prise ou administrations } \\
\text { Réf. : pas d'outil }\end{array}$} & ERP & 0,06 n.s. $(0,046)$ & 0,01 n.s. $(0,052)$ & 0,13 * $(0,077)$ \\
\hline & Outils de travail collaboratif & $0,43^{* \star *}(0,047)$ & $0,32^{\star \star \star}(0,055)$ & $0,31^{\star \star \star}(0,085)$ \\
\hline \multirow[t]{2}{*}{ Fonction } & Production, nettoyage, manutention & $-0,72^{* \star \star}(0,071)$ & $-0,49^{\star \star \star}(0,079)$ & - 0,13 n.s. $(0,120)$ \\
\hline & Comptabilité, gestion & $0,41^{\star \star \star}(0,079)$ & $0,16^{\star \star}(0,079)$ & $0,20 *(0,115)$ \\
\hline \multirow{2}{*}{$\begin{array}{l}\text { Réf. : secrétariat, saisie, } \\
\text { commercial }\end{array}$} & Études, R\&D, enseignement, soin & $-0,26^{\star \star \star}(0,083)$ & $-0,05$ n.s. $(0,087)$ & $0,23 *(0,122)$ \\
\hline & Autre fonction & $-0,28^{* * *}(0,078)$ & $-0,30^{* * *}(0,086)$ & 0,04 n.s. $(0,126)$ \\
\hline \multirow{3}{*}{$\begin{array}{l}\text { PCS } \\
\text { Réf. : employé }\end{array}$} & Cadres & $0,34^{\star \star \star}(0,077)$ & $0,40^{\star \star \star}(0,083)$ & $0,37^{\star \star \star}(0,121)$ \\
\hline & Professions intermédiaires & $0,47^{\star \star \star}(0,065)$ & $0,51^{\star \star \star}(0,070)$ & $0,25^{\star *}(0,106)$ \\
\hline & Ouvriers & $-0,89^{\star \star \star}(0,081)$ & $-0,73^{\star \star \star}(0,100)$ & $-0,98^{\star \star \star}(0,158)$ \\
\hline \multicolumn{5}{|l|}{ Sexe } \\
\hline Réf. : femme & Hommes & $-0,02$ n.s. $(0,049)$ & $-0,12^{\star \star}(0,054)$ & 0,03 n.s. $(0,080)$ \\
\hline \multirow{2}{*}{$\begin{array}{l}\text { Diplôme } \\
\text { Réf. : sans diplôme ou } 1^{\text {er }} \text { cycle }\end{array}$} & Supérieur au bac & 0,26 *** $(0,063)$ & 0,22 *** $(0,070)$ & $0,34^{\star \star \star}(0,104)$ \\
\hline & $2^{\mathrm{e}}$ cycle & $0,42^{\star \star *}(0,063)$ & $0,32^{\star \star \star}(0,071)$ & $0,41^{\star \star \star}(0,105)$ \\
\hline \multirow[t]{2}{*}{ Âge et ancienneté } & $\begin{array}{l}\text { Moins de } 40 \text { ans, ancienneté } \\
<15 \text { ans }\end{array}$ & $-0,76^{\star \star \star}(0,071)$ & $0,24^{\star \star \star}(0,080)$ & $-1,02^{\star \star \star}(0,107)$ \\
\hline & $\begin{array}{l}\text { Moins de } 40 \text { ans, ancienneté } \\
>=15 \text { ans }\end{array}$ & $-0,222^{\star \star}(0,103)$ & 0,17 n.s. $(0,120)$ & - 0,18 n.s. $(0,150)$ \\
\hline \multirow{3}{*}{$\begin{array}{l}\text { Réf. : } 50 \text { ans et plus, ancien- } \\
\text { neté supérieure à } 15 \text { ans }\end{array}$} & 40-49 ans, ancienneté $<15$ ans & $-0,74^{\star \star \star}(0,085)$ & 0,07 n.s. $(0,098)$ & $-0,93^{\star \star *}(0,143)$ \\
\hline & $40-49$ ans, ancienneté $>=15$ ans & - 0,07 n.s. $(0,074)$ & 0,12 n.s. $(0,085)$ & $-0,29^{* * *}(0,102)$ \\
\hline & $>=50$ ans, ancienneté $<15$ ans & $-0,65^{\star \star \star}(0,097)$ & $-0,17$ n.s. $(0,116)$ & $-0,74^{\star \star \star}(0,157)$ \\
\hline
\end{tabular}

Lecture : les modèles $4 M, 5 M$ et $6 M$ présentent les modélisations respectives des probabilités d'avoir cumulé des formations informatiques chez l'employeur actuel; d'avoir suivi une ou des formations à l'informatique en 2005 et 2006 ; et d'avoir suivi une formation à l'informatique d'au moins 30 heures sur l'ensemble du champ. La situation de référence est signalée dans la première colonne. Les coefficients sont issus de l'estimation des modèles Logit. Les écarts-types sont présentés entre parenthèses.

La probabilité d'avoir été formé à l'informatique en 2005-2006 est ainsi non significativement différente pour les salariés âgés de 50 ans et plus, que leur ancienneté soit inférieure ou supérieure à 15 ans.

n.s. non significatif ; ${ }^{* *}$ significatif au seuil de $1 \%$, ** significatif au seuil de $5 \%$, * significatif au seuil de $10 \%$.

Champ : fonction publique de l'État hors enseignants, magistrats, agents du ministère de la Défense et des établissements publics. Pour les secteurs marchands : salariés des entreprises marchandes non agricoles de plus de 20 salariés appartenant à un groupe ; utilisateurs de l'informatique.

Sources : COI 2006, Statistique publique, DGAFP-Dares-CEE. 
rents travaux. Au début de l'informatisation des entreprises, l'accès sélectif aux nouvelles technologies ouvrait des opportunités de carrière aux (jeunes) salariés qui s'étaient emparés de l'outil technique (Bonvin et Faguer, 2000 ; Gollac et Kramarz, 2000) ; elle se traduit désormais par la mise à l'écart d'une minorité de seniors.

Dès le début des années 1990, l'âge apparaissait moins comme un frein pour l'accès à l'informatique dans le secteur public que dans le privé (Moatty, 1995). En 2006, l'utilisation de l'informatique n'est pas liée à l'âge dans la fonction publique de l'État, alors que la part des plus de 50 ans y a fortement augmenté. Cet employeur dont la politique de gestion des ressources humaines repose sur la permanence de l'emploi serait davantage motivé à trouver des solutions pour permettre aux seniors à la fois l'accès à des postes informatisés et l'utilisation d'outils variés. Dans la fonction publique de l'État, les occasions d'être formé s'accroissent avec l'ancienneté, favorisant l'accès de l'informatique à tout âge. L'entraide, autre facteur favorable de l'utilisation professionnelle de l'informatique, peut également être interprétée comme l'un des effets de la permanence dans l'emploi et de la stabilité des collectifs.

Alors que le vieillissement de la population devrait encore s'accentuer dans les prochaines décennies, les implications des mécanismes de sélection par l'âge sont multiples, notamment concernant le financement et l'âge de la retraite. Aujourd'hui, moins d'un salarié sur deux est en emploi l'année qui précède la liquidation de son droit à la retraite, et si bien d'autres explications sont avancées (effets des dispositifs de prére-

Tableau 10

Formations à l'informatique selon des critères personnels et professionnels

Fonction publique de l'État

\begin{tabular}{|c|c|c|c|c|}
\hline & Modèle 4P & Modèle 5P & Modèle 6P \\
\hline & & $\begin{array}{l}\text { Cumul formation } \\
\text { informatique chez } \\
\text { l'employeur actuel }\end{array}$ & $\begin{array}{l}\text { Formation } \\
\text { informatique en } \\
2005-2006\end{array}$ & $\begin{array}{l}\text { Formation à } \\
\text { l'informatique } \\
\text { d'au moins 30h }\end{array}$ \\
\hline \multicolumn{2}{|l|}{ Constante } & $1,08^{\star \star \star}(0,271)$ & $-1,01^{\star \star \star}(0,260)$ & $-1,46^{\star \star \star}(0,301)$ \\
\hline \multirow{2}{*}{$\begin{array}{l}\text { Outils présents dans l'entre- } \\
\text { prise ou administrations } \\
\text { Réf. : pas d'outil }\end{array}$} & ERP & 0,01 n.s. $(0,153)$ & - 0,21 n.s. $(0,154)$ & 0,10 n.s. $(0,177)$ \\
\hline & Outils de travail collaboratif & 0,12 n.s. $(0,166)$ & 0,39 ** $(0,174)$ & 0,09 n.s. $(0,201)$ \\
\hline \multirow[t]{2}{*}{ Fonction } & Production, nettoyage, manutention & $-1,95^{\star \star \star}(0,278)$ & $-1,40 * \star *(0,314)$ & $-0,78^{\star \star}(0,363)$ \\
\hline & Comptabilité, gestion & - 0,26 n.s. $(0,263)$ & - 0,36 n.s. $(0,235)$ & - 0,25 n.s. $(0,277)$ \\
\hline \multirow{2}{*}{$\begin{array}{l}\text { Réf. : Secrétariat, saisie, } \\
\text { commercial }\end{array}$} & Études, R\&D, enseignement, soin & $-1,06^{\star \star \star}(0,295)$ & - 0,34 n.s. $(0,282)$ & - 0,36 n.s. $(0,336)$ \\
\hline & Autre fonction & $-0,69^{* \star \star}(0,256)$ & $-0,56^{\star \star}(0,245)$ & - 0,10 n.s. $(0,285)$ \\
\hline PCS & Cadres & $1,00^{\star \star \star}(0,263)$ & $0,94^{\star \star \star}(0,254)$ & 0,45 n.s. $(0,291)$ \\
\hline \multirow[t]{2}{*}{ Réf. : employé } & Professions intermédiaires & $0,78^{* \star *}(0,209)$ & 1,00 *** $(0,199)$ & 0,14 n.s. $(0,234)$ \\
\hline & Ouvriers & - 0,03 n.s. $(0,328)$ & - 0,11 n.s. $(0,454)$ & - 0,30 n.s. $(0,528)$ \\
\hline \multicolumn{5}{|l|}{ Sexe } \\
\hline Réf. : femme & Hommes & - 0,11 n.s. $(0,168)$ & - 0,20 n.s. $(0,171)$ & 0,19 n.s. $(0,200)$ \\
\hline \multirow{2}{*}{$\begin{array}{l}\text { Diplôme } \\
\text { Réf. : sans diplôme ou } 1^{\text {er }} \text { cycle }\end{array}$} & Supérieur au bac & - 0,02 n.s. $(0,226)$ & - 0,09 n.s. $(0,232)$ & 0,38 n.s. $(0,271)$ \\
\hline & $2^{\mathrm{e}}$ cycle & $0,71^{\star \star \star}(0,220)$ & 0,50 ** $(0,215)$ & 0,63 ** $(0,248)$ \\
\hline \multirow[t]{3}{*}{ Âge et ancienneté } & $\begin{array}{l}\text { Moins de } 40 \text { ans, ancienneté } \\
<15 \text { ans }\end{array}$ & $-0,58^{\star \star}(0,234)$ & 0,17 n.s. $(0,230)$ & $-1,33^{\star \star \star}(0,310)$ \\
\hline & $\begin{array}{l}\text { Moins de } 40 \text { ans, ancienneté } \\
>=15 \text { ans }\end{array}$ & $-0,80 *(0,456)$ & - 0,60 n.s. $(0,582)$ & - 0,81 n.s. $(0,644)$ \\
\hline & 40-49 ans, ancienneté $<15$ ans & $-0,82^{\star \star \star}(0,274)$ & - 0,29 n.s. $(0,295)$ & $-0,71^{\star \star}(0,339)$ \\
\hline \multirow{2}{*}{$\begin{array}{l}\text { Réf. : } 50 \text { ans et plus, ancien- } \\
\text { neté supérieure à } 15 \text { ans }\end{array}$} & $40-49$ ans, ancienneté $>=15$ ans & 0,12 n.s. $(0,222)$ & 0,19 n.s. $(0,203)$ & $-0,35$ n.s. $(0,224)$ \\
\hline & $>=50$ ans, ancienneté $<15$ ans & $-0,73^{\star \star \star}(0,257)$ & $-0,47^{*}(0,277)$ & $-0,60 * *(0,306)$ \\
\hline
\end{tabular}

Lecture : les modèles $4 P, 5 P$ et $6 P$ présentent les modélisations respectives des probabilités d'avoir cumulé des formations informatiques chez l'employeur actuel; d'avoir suivi une ou des formations à l'informatique en 2005 et 2006 ; et d'avoir suivi une formation à l'informatique d'au moins 30 heures dans la fonction publique de l'État. La situation de référence est signalée dans la première colonne. Les coefficients sont issus de l'estimation des modèles Logit. Les écarts-types sont présentés entre parenthèses.

La probabilité d'avoir été formé à l'informatique en 2005-2006 des agents de 50 ans et plus dont l'ancienneté est inférieure à 15 ans est ainsi significativement inférieure à celle des agents de 50 ans et plus dont l'ancienneté est supérieure à 15 ans.

Champ : fonction publique de l'État hors enseignants, magistrats, agents du ministère de la Défense et des établissements publics, utilisateurs de l'informatique.

Sources : COI 2006, statistique publique, DGAFP-Dares-CEE. 


\section{Graphique VII \\ Entraide et utilisation professionnelle de l'informatique selon l'âge}

Score d'entraide

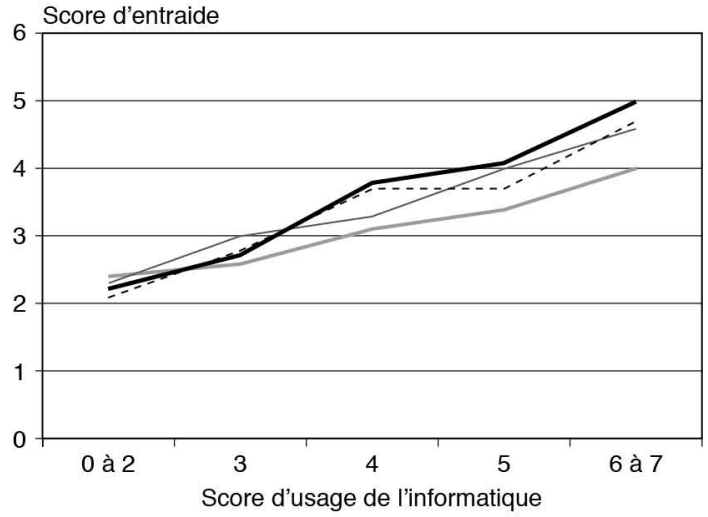

- -30 ans $-30-39$ ans $---40-49$ ans -50 ans et +

Lecture : les salariés de moins de 30 ans ayant un score d'usage de l'informatique le plus élevé (de 6 ou 7) ont un score d'entraide de 4. Pour les salariés âgés de 50 ans et plus dont le score d'usage de l'informatique est également le plus élevé, le score d'entraide est de 5.

Champ : fonction publique de l'État hors enseignants, magistrats, agents du ministère de la Défense et des établissements publics. Pour les secteurs marchands : salariés des entreprises marchandes non agricoles de plus de 20 salariés.

Sources : COI 2006, statistique publique, DGAFP-Dares-CEE, données pondérées. traite, dégradation de la santé...), les changements technologiques sont une des causes possibles de cette éviction de l'emploi des salariés vieillissants (Blanchet, 2007).

Pourtant, comme nos résultats le montrent, il n'y pas d'âge à partir duquel on serait moins capable d'utiliser l'informatique dès lors que la nature de l'activité exercée le requiert, que l'entraide entre salariés est encouragée et que des politiques de formation adéquates sont mises en place. Dans la fonction publique de l'État en tous cas, le vieillissement des salariés n'est pas un obstacle à l'utilisation professionnelle généralisée de l'informatique.

\section{BIBLIOGRAPHIE}

Alcaud D. et Lakel A. (2004), « Les nouveaux « visages » de l'administration sur Internet : pour une évaluation des sites publics de l'État ", Revue Française d'Administration Publique, $\mathrm{n}^{\circ} 110$, p. 297-313.

Ananian S. et Aubert P. (2006), « Travailleurs âgés, nouvelles technologies et changements organisationnels », Économie et Statistique, n ${ }^{\circ} 397$.

Aubert P., Caroli E. et Roger M. (2006), " Nouvelles technologies et nouvelles formes d'organisation du travail : quelles conséquences pour l'emploi des salariés âgés ? ", Revue économique, vol. $57, \mathrm{n}^{\circ} 6$, pp. 1329-1349.

Baëhr A. (2008), «Vieillissement dans la fonction publique : des agents plus âgés à l'Ouest, au Sud et chez les cadres, plus jeunes à l'hôpital et chez les enseignants », Point Stat $\mathrm{n}^{\circ}$ 05, DGAFP.

Behaghel L., Caroli E. et Roger M. (2009), « Départ des travailleurs âgés, formation continue dans les entreprises innovantes », École d'économie de Paris, Working paper, $\mathrm{n}^{\circ}$ 2009-50.
Behaghel L. et Gautié J. (2006), « From internal to transitional labour markets ? Firms restructuring and early retirement in France », Document de travail, $\mathrm{CEE}, \mathrm{n}^{\circ} 57$.

Besnard S., Chevalier P., Guillemot D., Kocoglu Y. et Victor P. (2007) « Des Tic de plus en plus diversifiés dans les entreprises », Insee Première, $n^{\circ} 1126$.

Bezes P. (2009), Réinventer l'État. Les réformes de l'administration française (1962-2008), Paris, PUF.

Blanchet D. (2007), "Santé, vieillissement et retraites en Europe ", Économie et Statistique, $n^{\circ} 403-404$.

Blasco S. Lê J. et Monso O. (2009), « Formation continue en entreprise et promotion sociale, mythe ou réalité ? », Formations et emploi, Insee références, pp. 27-42.

Bonvin F. et Faguer J.-P. (2000), « Une génération d'autodidactes ", Actes de la recherche en sciences sociales, $\mathrm{n}^{\circ} 134$, pp. 76-83. 
Cézard M., Gollac M. et Rougerie C. (2000), «L'ordinateur, outil de travail et bien culturel » Actes de la recherche en sciences sociales, $\mathrm{n}^{\circ} 134$, pp. 22-28.

Caroli E. (2000), « Flexibilité interne versus flexibilité externe du travail : quels enseignements peut-on tirer de l'approche de la firme en termes de compétences ? ", Document de travail LEAInra, $\mathrm{n}^{\circ} 00-10$.

Delgoulet C. et Gonon O. (2000), « L'épreuve de l'apprentissage en milieu de carrière », Formation emploi, $\mathrm{n}^{\circ}$ 71, pp. 53-65.

DGAFP (2009), Rapport annuel sur l'état de la fonction publique, vol. 1, Faits et chiffres 20082009, Paris, La Documentation française.

Diaye M.-A., Greenan N., Minni C. et Marques S.-R. (2006), « Renouvellement des générations, précarité de l'emploi des jeunes et dynamique technologique des entreprises », Revue Économique, vol. 57, n० 6, pp. 1295-1325.

Dodier N. (1995), Les hommes et les machines : la conscience collective dans les sociétés technicisées, Paris, Métailié.

Dodier N. (1997), « Remarques sur la conscience $\mathrm{du}$ collectif dans les réseaux sociotechniques », Sociologie du Travail, $\mathrm{n}^{\circ}$ 2, p. 131-148.

Friedberg L. (2003), " The Impact of Technological Change on Older Workers : Evidence from Data on Computer ", Industrial and Labor Relations Review, vol. 56, n³, pp. 511-529.

Gaudart C. (2000), «Quand l'écran masque 1'expérience des opérateurs vieillissants : changement de logiciel et activité de travail dans un organisme de services », Pistes, vol. 2, n 2 .

Gautié J. (2002), « Déstabilisation des marchés internes et gestion des âges sur les marchés du travail », Document de travail du Centre d'Études de l'Emploi, $\mathrm{n}^{\circ} 15$.

Gautier L., Jean O. et Reynaud D. (2009), « Les départs à la retraite dans les trois fonctions publiques », Rapport annuel sur l'État de la fonction publique, vol. 1 : Faits et chiffres, DGAFP.

Gollac M. (1996), « Le capital est dans le réseau : La coopération dans l'usage de l'informatique », Travail et Emploi, $\mathrm{n}^{\circ} 68$, pp. 39-60.

Gollac M. et Volkoff S. (2000), Les conditions de travail, La Découverte, Paris.
Gollac M., Greenan N. et Hamon-Cholet S. (2000), « L'informatisation de l'ancienne économie : nouvelles machines, nouvelles organisations et nouveaux travailleurs ", Économie et statistique, $\mathrm{n}^{\circ} 339-340, \mathrm{pp}$. 171-201.

Gollac M. et Kramarz F. (2000), « L'informatique, comme pratique et comme croyance », Actes de la recherche en sciences sociales, $\mathrm{n}^{\circ} 134$.

Guillemot D. (2008), « Une fonction publique informatisée », Connaissance de l'emploi, $\mathrm{n}^{\circ} 57$.

Guillemot D. et Peyrin A. (2009), « L'informatisation dans les ministères en $2006 »$, Point stat, $\mathrm{n}^{\circ} 12$, DGAFP.

Guillemot D. et Peyrin A. (2008), Les agents de la fonction publique de l'État, des travailleurs comme les autres? Une exploration de l'enquête Changements organisationnels et informatisation 2006, Rapport de recherche du Centre d'Études de l'Emploi (CEE) à la DGAFP.

Hamon-Cholet S. et Vinck L. (2004), « Les NTIC, des technologies banalisées ? », in Bué J., Coutrot T. et Puech I., Conditions de travail, les enseignements de 20 ans d'enquête, Octarès.

Lainé F. (2003), « Les seniors et la formation continue : un accès en général limité mais avec de grandes différences selon les situations professionnelles », Premières synthèses, ${ }^{\circ}{ }^{\circ} 121$, Dares.

Lau E. (2004), « Principaux enjeux de l'administration électronique dans les pays membres de l'OCDE », Revue Française d'Administration Publique, $\mathrm{n}^{\circ} 110$, vol. 2, pp. 225-43.

Levasseur S. (2008), « Progrès technologique et employabilité des seniors ", Revue de l'OFCE, vol. $3, \mathrm{n}^{\circ} 106$, pp. 155-184.

Marquié J.-C. et Bacarat B. (1992), « Technologies nouvelles et travailleurs anciens : le cas de l'informatique de bureau », Travail et emploi, $\mathrm{n}^{\circ} 54$, pp. 34-49.

Minni C. et Topiol A. (2003), « Les entreprises face au vieillissement de leurs effectifs », Économie et statistique, ${ }^{\circ} 368$, pp. 43-63.

Moatty F. (1993), « Qui utilise l'informatique au travail ? », Dossier de recherche, CEE.

Moatty F. (1995), « Public, privé, la diversité des critères d'accès à l'informatique ", Revue française des affaires sociales, $\mathrm{n}^{\circ} 1$. 
Moatty F. (2009), « Le poids du niveau d'éducation dans l'accès à l'informatique », in L'évolution des cultures numériques, de la mutation du lien social à l'organisation du travail (Licoppe dir.) Ed FYP, pp. 104-109.

Perez C. (2002), « La formation continue des agents de la fonction publique », Bref, $\mathrm{n}^{\circ} 184$, Cereq.

Rouquette C. (1999), « L'informatique : une technique assimilée par les jeunes générations », Insee Première ${ }^{\circ} 643$.
Volkoff S. et Cau-Bareille D. (1998), « Vieillissement et informatisation dans le tertiaire ? Une approche par l'analyse de l'activité de travail », Travail et Emploi, $\mathrm{n}^{\circ} 76$.

Volkoff S., Laville A. et Maillard M.-C. (1992), «Âge et travail : contraintes, sélection et difficultés chez les 40-50 ans. Une analyse de l'enquête ESTEV », Travail et emploi, $\mathrm{n}^{\circ}$ 54, pp. 20-33. 


\section{CALCUL D'UN SCORE D'UTILISATION DE L'INFORMATIQUE}

On cherche à construire une échelle d'utilisation professionnelle de l'informatique qui rende compte d'une progression dans l'utilisation de l'outil, d'un usage simple ou basique (saisie, consultation d'information dans une base de donnée professionnelle...) à un usage varié, multipliant les possibilités offertes par l'outil (utilisation du mèl, accès à internet, intranet, accès à distance au système d'information de l'entreprise...). Pour cela, on construit un score simple en additionnant les valeurs attribuées aux modalités de cinq variables. Ces variables sont choisies parmi les plus fortement contributives sur les deux premiers axes d'une analyse des correspondances multiples portant sur 12 variables de l'enquête $\mathrm{COI} 2006$ retraçant les utilisations professionnelles de l'informatique (Guillemot et Peyrin, 2008). On vérifie que le travail de saisie est surreprésenté dans les scores les moins élevés.

- Combien de temps le salarié passe-t-il chaque jour à traiter ses messages électroniques professionnels?

0 : salarié non concerné (pas de boîte aux lettres électronique) ;

1 : moins de 30 minutes ;

$2: 30$ minutes et plus.

- Quelle est l'étendue de son accès professionnel à Internet?

0 : salarié non concerné (aucun accès) ;

1 : à une liste limitée de sites ;

2 : à toute la toile (sauf éventuellement certains sites interdits).
- Le salarié utilise-t-il un intranet ou un autre réseau de communication interne?

0 : non ;

1 : oui.

- Le salarié renseigne-t-il en ligne des demandes de congés ou d'autres formulaires administratifs de son entreprise?

0 : non ;

1 : oui.

- Le salarié utilise-t-il professionnellement un micro-ordinateur pour des activités professionnelles en dehors de son bureau?

0 : salarié non concerné (n'utilise jamais de matériel de l'entreprise en dehors) ;

1 : oui, utilise un micro-ordinateur en dehors des murs de l'entreprise ;

2 : oui, et a en plus accès au système d'information de son entreprise à distance.

Le score est constitué de la somme des modalités, 0 traduisant l'absence d'usage professionnel de l'informatique, 9 une utilisation cumulant des formes variées, à la fois informationnelles, communicationnelles et nomades. Pour plus de lisibilité, les neuf positions sont regroupées en quatre modalités : pas d'utilisation (0), utilisation basique (1 à 3), utilisation intermédiaire (4 à 6) et utilisation variée (7 à 9$)$. 


\section{MESURES DE L'ENTRAIDE}

Comme pour le score mesurant la maîtrise de l'informatique au travail, on a sélectionné les sept variables les plus contributives aux deux premiers axes d'une analyse des correspondances multiples (ACM) construite à partir de 12 variables de l'enquête COI 2006 (Guillemot et Peyrin, 2008) :

- Le salarié a-t-il un nombre de collègues supérieur ou égal à 10 ?

$0:$ non ;

1 : oui.

- Le salarié reçoit-il de l'aide de la part de ses collègues en cas de surcroît de travail ou lorsqu'il affronte une tâche délicate?

0 : non;

1 : oui.

- Le salarié reçoit-il de l'aide de la part de son chef en cas de surcroît de travail ou lorsqu'il affronte une tâche délicate?

$0:$ non ;

1 : oui.

- Le salarié reçoit-il de l'aide de la part d'un autre salarié de l'entreprise en cas de surcroît de travail ou lorsqu'il affronte une tâche délicate?

0 : non ;

1 : oui.
- Le salarié a-t-il de fréquentes occasions de discuter avec ses collègues?

0 : non ;

1 : oui.

- Le salarié juge-t-il bonne l'ambiance de travail avec ses collègues?

0 : non ;

1 : oui.

- Le salarié a-t-il demandé de l'aide dans au moins un des cas suivants : au fonctionnement d'un matériel ou logiciel complexe ; au déroulement d'une procédure complexe ; à l'attitude vis à vis du public ; à d'autres aspects du travail ?

0 : non ;

1 : oui.

Quel que soit l'âge, la plupart des modes d'entraide tend à accroître la maîtrise d'une informatique professionnelle avancée (cf. graphique). Les scores des seniors associés à la taille du collectif, la formation sur le tas de la part des collègues, les discussions, l'aide reçue du chef ou de salariés de l'entreprise en cas de travail délicat et la bonne ambiance sont nettement supérieurs à ceux des plus jeunes. Les seniors font probablement davantage appel à l'entraide pour pallier des lacunes ou un manque de pratique dans les aspects les plus complexes de l'informatique. 
Graphique

Score moyen d'utilisation de l'informatique par type d'entraide et âge

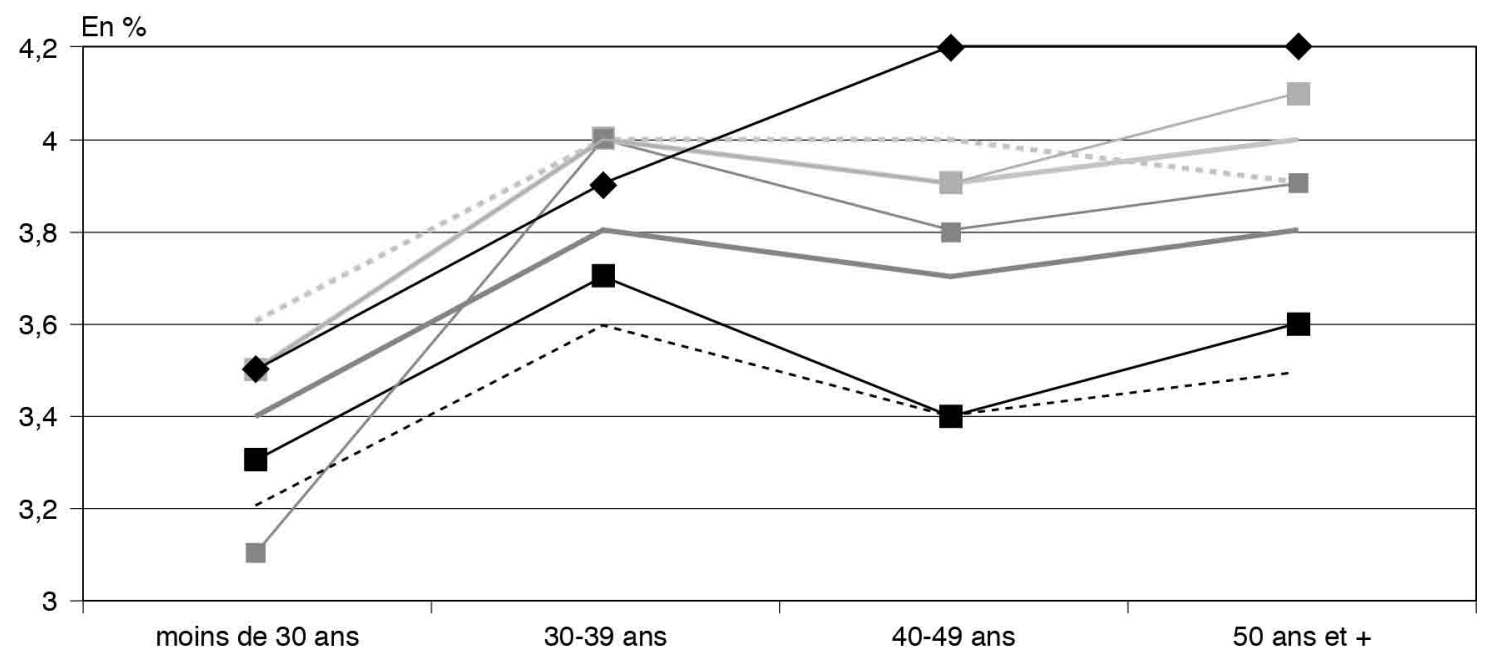

Au moins 10 collègues
Aide du chef en cas de travail délicat

Lecture : le score moyen d'entraide des salariés dont le collectif comprend au moins dix collègues est de 3,5 pour les salariés âgés de moins de trente ans, contre 4,2 pour les salariés âgés de 50 ans et plus.

Champ : fonction publique de l'Etat hors enseignants, magistrats, agents du ministère de la Défense et des établissements publics. Pour les secteurs marchands : salariés des entreprises marchandes non agricoles de plus de 20 salariés.

Sources : COI 2006, Statistique publique, DGAFP-Dares-CEE. 